\title{
Some numerical applications of generalized Bernstein operators
}

\author{
Donatella Occorsio, Maria Grazia Russo*, AND Woula Themistoclakis
}

\begin{abstract}
In this paper, some recent applications of the so-called Generalized Bernstein polynomials are collected. This polynomial sequence is constructed by means of the samples of a continuous function $f$ on equispaced points of $[0,1]$ and depends on an additional parameter which can be suitable chosen in order to improve the rate of convergence to the function $f$, as the smoothness of $f$ increases, overcoming the well-known low degree of approximation achieved by the classical Bernstein polynomials or by the piecewise polynomial approximation. The applications considered here deal with the numerical integration and the simultaneous approximation. Quadrature rules on equidistant nodes of $[0,1]$ are studied for the numerical computation of ordinary integrals in one or two dimensions, and usefully employed in Nyström methods for solving Fredholm integral equations. Moreover, the simultaneous approximation of the Hilbert transform and its derivative (the Hadamard transform) is illustrated. For all the applications, some numerical details are given in addition to the error estimates, and the proposed approximation methods have been implemented providing numerical tests which confirm the theoretical estimates. Some open problems are also introduced.
\end{abstract}

Keywords: Bernstein polynomials, approximation by polynomials, numerical integration on uniform grids, Fredholm integral equations on uniform grids.

2020 Mathematics Subject Classification: 41A10, 65D32, 65R10, 65R20.

Dedicated to Professor Francesco Altomare, on occasion of his 70th birthday, with esteem and friendship.

\section{INTRODUCTION}

Bernstein polynomials $B_{m} f$ constitute a classical approximation of a continuous function $f$ based on the samples of $f$ at equidistant nodes of $[0,1]$. They have been widely studied in literature (see for instance [23], [3]) and provide a constructive proof of the Weierstrass theorem, since the positive Bernstein operators $B_{m}: f \rightarrow B_{m} f$ fits the assumptions of the Korovkin theorem (see e.g. [2]).

On the other hand, in many applications, the available data are often the values of the target function at equally spaced point sets, which would make suitable to apply the Bernstein polynomials. However, such polynomials are rarely used in the numerical approximation because a rate of convergence faster than $\frac{1}{m}$ cannot be obtained for more regular functions than absolutely continuous functions $f$ s.t. $\left\|f^{\prime \prime} \varphi^{2}\right\|<\infty$, where $\varphi(x)=\sqrt{x(1-x)}$ (see e.g. [13]).

In order to get an higher rate of approximation, independently Micchelli [30], Felbeker [17] and Mastroianni-Occorsio [24] introduced and studied the following combinations of iterates

Received: 25.01.2021; Accepted: 12.02.2021; Published Online: 01.03.2021

*Corresponding author: Maria Grazia Russo; mariagrazia.russo@unibas.it

DOI: $10.33205 / \mathrm{cma} .868272$ 
of the Bernstein operator $B_{m}$

$$
B_{m, s}=I-\left(I-B_{m}\right)^{s}=\sum_{i=1}^{s}\left(\begin{array}{l}
s \\
i
\end{array}\right)(-1)^{i-1} B_{m}^{i}, \quad s \in \mathbb{N}
$$

where $B_{m}^{i}=B_{m}\left(B_{m}^{i-1}\right), i \geq 1, B_{m}^{0}=I$ and $I$ is the identity operator.

Similarly to $B_{m}$, for all $s \in \mathbb{N}$, the operators $B_{m, s}$ map continuous functions $f$ into polynomials of degree $m$. The polynomials $B_{m, s} f$ are known in the literature as Generalized Bernstein polynomials of parameter $s$ (shortly GBs polynomials). Like $B_{m} f$, they require the samples of $f$ at the $m+1$ equispaced points of $[0,1]$ and interpolate $f$ at the extremes. However, differently from the "originating" Bernstein operator, GBs operators are not always positive, as it can be clearly expected, since as $m \in \mathbb{N}$ is fixed and $s \rightarrow \infty$, we have that $B_{m, s} f \rightarrow L_{m} f$ uniformly in $[0,1]$, where $L_{m} f$ is the Lagrange polynomial interpolating $f$ at the same equispaced nodes [24].

Nonetheless, for any fixed $s \in \mathbb{N}$ and $m \rightarrow \infty$, we have that $B_{m, s} f \rightarrow f$ uniformly in $[0,1]$ and suitable choices of the additional parameter $s>1$, allow to increase the approximation rate achieved by the classical Bernstein polynomials, being $m^{-s}$ the saturation order of $B_{m, s}$ [30]. In addition, as main property and, in some sense, cornerstone of the study, in [30, 24] the authors independently stated how the rate of convergence in approximating $f$ improves as the smoothness of $f$ increases. To be more precise, they proved that any function $f \in C^{2 s}([0,1])$ can be uniformly approximated by the sequence $\left\{B_{m, s} f\right\}_{m \in \mathbb{N}}$ with the rate of convergence $\mathcal{O}\left(m^{-s}\right)$. A more refined error estimate was proven in [19] by using the Ditzian-Totik $\varphi-$ modulus of $f$ having order $2 s$. GBs polynomials were further investigated from other many authors and from many different points of view (see e.g. [4], [36], [38], [15], [8], [10], [34], [35]). In particular, a short history of GBs polynomials can be found in [19], with a wide bibliography on the topic.

The aim of the present paper is to "promote" GBs approximation in the applications by collecting some numerical methods based on GBs polynomials, which show how these polynomials may be useful from the applicative point of view.

It is in fact known that in many applications the samples of $f$ are sometimes obtained by devices, or by measures detected at equidistant times. Such an experimental nature of the data precludes the use of those global techniques of approximation that have optimal performance, but are based on specified (non uniform) distribution of nodes such as the zeros of orthogonal polynomials.

On the contrary, GBs polynomials require data at equally spaced points and, differently from piecewise polynomials or ordinary Bernstein approximations, as the smoothness of $f$ increases, suitable choices of $s$ allow to improve the rate of convergence. For this reason, GBs polynomials might be fruitfully used in the applications, where the data are taken at equidistant nodes.

In particular, they have been successfully employed in some applications that we will summarize in this paper, where we provide some improvements of the already known results.

The numerical quadrature of ordinary integrals on the interval $[0,1]$ and on the square $[0,1]^{2}$, the numerical solution of Fredholm Integral Equations of the second kind (FIEs) on such domains, and, finally, the numerical computation of the finite Hilbert and Hadamard transforms on $[0,1]$ are the applications we will deal with.

FIEs play an important role in various fields of the applied sciences, since they model many problems in elasticity, fluid-dynamics, etc. . Also, the Hilbert transform is widely used for applications in several fields. Among them, there are partial differential equations, optics (X-ray crystallography, electron-atom scattering), electrodynamics and quantum mechanics 
(Kramers-Kronig relation), signal processing (phase retrieval, transfer functions of linear systems, spectral factorization) (see e.g. [22]). Moreover, both the Hilbert and Hadamard transforms, the latter regarded as the first derivative of the Hilbert one, are widely used to formulate boundary-value problems in many areas of mathematical physics (potential theory, fracture mechanics, aerodynamics, elasticity, etc.) in terms of singular integral equations in $(0,1)$ (see e.g. [21, 27, 28, 29, 37] and the references therein). Hence, numerical methods based on GBs for the above applications can be applied in different contexts whenever the discrete data are available at equally spaced nodes.

We recall that in $[32,33]$ stable and convergent quadrature and cubature rules have been obtained by replacing the integrand by GBs polynomials in one and in two variables. Based on these rules, in the same papers, Nyström methods have been proposed for the numerical solution of one and two-dimensional FIEs. Studying such equations in Sobolev type spaces, the authors proved that in both the dimensions the methods are numerically stable, convergent and the involved linear systems are well-conditioned. Here, we extend the results given in [32] by providing error estimates in the wider class of Hölder-Zygmund spaces. Moreover, in the bivariate case, we consider the tensor product of GBs operators using different values for both the degrees and the parameters. In this way, we get a more flexible approximation tool than that proposed in [33].

The approximation of the Hilbert transform in $(0,1)$, by means of GBs polynomials, was firstly investigated in $[25,26]$. Such idea has been recently revised in [18], where the simultaneous approximation of the Hilbert transform and its first derivative was proposed in $(-1,1)$.

In this paper, following the ideas in [18], we construct quadrature rules for both the Hilbert and Hadamard transforms in $(0,1)$, by means of a shrewd use of the simultaneous approximation by GBs polynomials. Such approach allows to approximate both the integral transforms, by using the same samples of $f$ at a grid of equally spaced nodes. Moreover, as in [18], some improvements from both the theoretical and computational point of view, are achieved w.r.t. those shown in $[25,26]$. We determine weighted pointwise estimates of the quadrature errors, in the general case of density functions satisfying the Dini-type conditions involving the DitzianTotik moduli of smoothness [13], as well as in the case of smoother functions in Sobolev and Hölder-Zygmund spaces. Concerning the numerical computation, recurrence relations for the quadrature coefficients in $[0,1]$ are given either for the Hilbert and the Hadamard transforms. Moreover, such new recurrence relations preserve the more stable Bernstein polynomial basis and do not require the transformation into the basis $\left\{1, x, \ldots, x^{m}\right\}$ as done in [25, 26].

For all the applications, we give some numerical tests and graphs in order to confirm the theoretical results and to show some numerical evidences on the role of the involved parameters $m$ and $s$ and the interaction between them.

Finally along the paper, the reader can find some open problems that could be interesting for further investigations.

The outline of the paper is as follows. Section 2 contains some notation and preliminary results about the approximation tools and the functional spaces. Section 3 is devoted to the GBs polynomials, their properties and the convergence results also for the simultaneous approximation. In Section 4, the quadrature formula based on GBs approximation is proposed. Section 5 is devoted to the Nyström method based on the quadrature rule of Section 4 . Section 6 contains the results on the simultaneous approximation of the Hilbert and Hadamard transforms. Section 7 shows some recent results in the bivariate case. Finally, Section 8 includes some computational details, that describe the practical implementation of the formulae based on GBs polynomials, and used in the paper. The tests given for each application were performed in double precision arithmetic. 


\section{NOTATION AND PRELIMINARY RESULTS}

In the sequel, $\mathcal{C}$ will denote a generic positive constant which may differ at different occurrences and $\mathcal{C} \neq \mathcal{C}(a, b, .$.$) indicates that \mathcal{C}$ is independent of $a, b, \ldots$. Moreover, if $A, B>0$ depend on some parameters the notation $A \sim B$ means that there are fixed constants $\mathcal{C}_{1}, \mathcal{C}_{2}>0$ (independent of the parameters in $A, B$ ) such that $\mathcal{C}_{1} A \leq B \leq \mathcal{C}_{2} A$.

For any integer $m \geq 0$, we set $N_{0}^{m}:=\{0,1,2, \ldots, m\}$ and denote by $\mathbb{P}_{m}$ the set of all algebraic polynomials of degree at most $m$. In the Banach space $C^{0}([0,1])$ of the continuous functions on $[0,1]$ endowed with the uniform norm $\|f\|:=\max _{x \in[0,1]}|f(x)|$, the error of best approximation of $f \in C^{0}([0,1])$ in $\mathbb{P}_{m}$ is defined as

$$
E_{m}(f)=\min _{P \in \mathbb{P}_{m}}\|f-P\|
$$

and the Weierstrass theorem ensures that

$$
f \in C^{0}([0,1]) \Longleftrightarrow \lim _{m \rightarrow \infty} E_{m}(f)=0 .
$$

A constructive proof of this result is given by the well-known Bernstein polynomials

$$
B_{m} f(x)=\sum_{k=0}^{m} p_{m, k}(x) f\left(t_{k}\right), \quad t_{k}:=\frac{k}{m}, \quad x \in[0,1], \quad m \geq 1,
$$

where

$$
p_{m, k}(x)=\left(\begin{array}{c}
m \\
k
\end{array}\right) x^{k}(1-x)^{m-k}, \quad k \in N_{0}^{m}, \quad x \in[0,1],
$$

are the fundamental Bernstein polynomials of degree $m$, which satisfy the following recurrence relation

$$
p_{m, k}(x)=(1-x) p_{m-1, k}(x)+x p_{m-1, k-1}(x), \quad k \in N_{0}^{m}, \quad m \geq 1,
$$

being $p_{m, k}(x) \equiv 0$, for $k \notin N_{0}^{m}$.

It is well-known that, for all $f \in C^{2}([0,1])$ and $m \in \mathbb{N}$ sufficiently large, Bernstein polynomials satisfy

$$
\left\|f-B_{m} f\right\| \leq \frac{\mathcal{C}}{m}, \quad \mathcal{C} \neq \mathcal{C}(m)
$$

and that the convergence rate does not improve by increasing the smoothness of $f$ as, instead, it happens for $E_{m}(f)$.

A useful tool to measure the smoothness of $f \in C^{0}([0,1])$ is the following Ditzian-Totik modulus of smoothness [13, (2.1.2)]

$$
\omega_{\varphi}^{r}(f, t)=\sup _{0<h \leq t}\left\|\Delta_{h \varphi}^{r} f\right\|, \quad r \in \mathbb{N},
$$

defined by means of the following finite differences with variable step-size

$$
\Delta_{h \varphi(x)}^{r} f(x)=\sum_{k=0}^{r}(-1)^{k}\left(\begin{array}{l}
r \\
k
\end{array}\right) f\left(x+(r-2 k) \frac{h}{2} \varphi(x)\right),
$$

where throughout the paper, it is $\varphi(x):=\sqrt{x(1-x)}$ and $x \in[0,1]$.

Denoting by $A C_{L o c}$ the space of all locally absolutely continuous functions on [0,1] (i.e. which are absolutely continuous in every closed subinterval $[a, b]$ in $(0,1))$, such modulus can be estimated by means of the following equivalent $K$-functional [13, Th. 2.1.1]

$$
\omega_{\varphi}^{r}(f, t) \sim K_{r, \varphi}\left(f, t^{r}\right):=\inf \left\{\|f-g\|+t^{r}\left\|g^{(r)} \varphi^{r}\right\|: g^{(r-1)} \in A C_{L o c}\right\} .
$$


Similarly to the classical modulus of smoothness given by

$$
\begin{gathered}
\omega^{r}(f, t)=\sup _{0<h \leq t}\left\|\Delta_{h}^{r} f\right\|, \quad r \in \mathbb{N}, \\
\omega^{r}(f, t) \sim K_{r}\left(f, t^{r}\right):=\inf \left\{\|f-g\|+t^{r}\left\|g^{(r)}\right\|: g^{(r-1)} \in A C_{L o c}\right\},
\end{gathered}
$$

we have

$$
\lim _{t \rightarrow 0} \omega_{\varphi}^{r}(f, t)=0, \quad \forall f \in C^{0}([0,1]) .
$$

Nevertheless, by taking the variable step-size $h \varphi(x)$, that decreases more and more as $x$ approaches to the extremes of $[0,1]$, the Ditzian-Totik modulus better describes the behaviour of the polynomial approximation close to the endpoints. In fact, the following Jackson and Stechkin-type inequalities hold true [13, Th. 7.2.1 and Th. 7.2.4]

$$
\begin{aligned}
E_{m}(f) & \leq \mathcal{C} \omega_{\varphi}^{r}\left(f, \frac{1}{m}\right), \quad \forall r<m, \quad \mathcal{C} \neq \mathcal{C}(m, f), \\
\omega_{\varphi}^{r}(f, t) & \leq \mathcal{C} t^{r} \sum_{0 \leq k \leq 1 / t}(1+k)^{r-1} E_{k}(f), \quad \mathcal{C} \neq \mathcal{C}(t, f)
\end{aligned}
$$

and these direct and converse results yield [13, Corollary 7.2.5]

$$
E_{m}(f)=\mathcal{O}\left(m^{-r}\right) \Longleftrightarrow \omega_{\varphi}^{k}(f, t)=\mathcal{O}\left(t^{r}\right), \quad k>r>0 .
$$

We point out that the implication " $\Longrightarrow$ " does not hold for the classical moduli, that are related to the Ditzian-Totik ones as follows

$$
\omega_{\varphi}^{r}(f, t) \leq \mathcal{C} \omega^{r}(f, t), \quad \mathcal{C} \neq \mathcal{C}(f, t), \quad r \in \mathbb{N} .
$$

Now, let us consider the following Sobolev-type spaces

$$
W_{r}=\left\{f \in C^{0}([0,1]): f^{(r-1)} \in A C_{L o c},\left\|f^{(r)} \varphi^{r}\right\|<\infty\right\}, \quad r \in \mathbb{N},
$$

equipped with the norm $\|f\|_{W_{r}}:=\|f\|+\left\|f^{(r)} \varphi^{r}\right\|$. By virtue of the previous results, the following properties hold for all $f \in W_{r}$ and $r \in \mathbb{N}$,

$$
\begin{aligned}
E_{m}(f) & \leq \frac{\mathcal{C}}{m^{r}}, \quad \mathcal{C} \neq \mathcal{C}(m), \\
\omega_{\varphi}^{k}(f, t) & \leq \mathcal{C} t^{r}\left\|f^{(r)} \varphi^{r}\right\|, \quad \forall k \geq r, \quad \mathcal{C} \neq \mathcal{C}(t, f), \\
\omega_{\varphi}^{r}(f, t) & =o\left(t^{r}\right) \Longrightarrow f \in \mathbb{P}_{r-1} .
\end{aligned}
$$

Denoting by $C^{k}([0,1])$ the space of all continuously differentiable functions till the order $k \in \mathbb{N}$, we also recall that classical moduli satisfy

$$
\omega^{k}(f, t) \leq \mathcal{C} t^{r}\left\|f^{(r)}\right\|, \quad \forall f \in C^{r}([0,1]), \quad \forall k \geq r, \quad \mathcal{C} \neq \mathcal{C}(t, f) .
$$

The Hölder-Zygmund type spaces based on Ditzian-Totik modulus of smoothness are defined as follows

$$
Z_{\lambda}=\left\{f \in C^{0}: \quad \sup _{t>0} \frac{\omega_{\varphi}^{r}(f, t)}{t^{\lambda}}<\infty, \quad r>\lambda\right\}, \quad \forall \lambda>0
$$

and equipped with the norm

$$
\|f\|_{Z_{\lambda}}=\|f\|+\sup _{t>0} \frac{\omega_{\varphi}^{r}(f, t)}{t^{\lambda}}, \quad r>\lambda .
$$


For any $\lambda>0$, the space $Z_{\lambda}$ constitutes a particular case of the Besov-type spaces studied in [14] and in the case $\lambda=r \in \mathbb{N}$ and the previously introduced Sobolev space $W_{r}$ in continuously imbedded in $Z_{r}$. More generally, it has been proved that [14, Theorem 2.1]

$$
\|f\|_{Z_{\lambda}} \sim\|f\|+\sup _{n>0}(n+1)^{\lambda} E_{n}(f), \quad \forall \lambda>0 .
$$

Such equivalence ensures that the definitions (2.13) and (2.14) are indeed independent of the integer $r>\lambda$ we choose. Moreover, (2.15) yields the following characterization of the continuous functions $f \in Z_{\lambda}$

$$
f \in Z_{\lambda} \Longleftrightarrow E_{n}(f)=\mathcal{O}\left(n^{-\lambda}\right), \quad \forall \lambda>0 .
$$

In particular, for all $f \in Z_{\lambda}$ and any $r>\lambda>0$, we get

$$
\omega_{\varphi}^{r}(f, t) \leq \mathcal{C} t^{\lambda}\|f\|_{Z_{\lambda}}, \quad \mathcal{C} \neq \mathcal{C}(f, t) .
$$

\section{THE GENERALIZED BERNSTEIN POLYNOMIALS}

Let $f \in C^{0}([0,1])$ and

$$
B_{m}^{i} f(x):=B_{m}\left(B_{m}^{i-1} f\right)(x), \quad B_{m}^{0} f:=f, \quad m, i \in \mathbb{N}
$$

be the $i$-th iterate of the Bernstein polynomial (2.2). Fixed an integer parameter $s \geq 1$, the Generalized Bernstein polynomial of parameter $s$ and degree $m \in \mathbb{N}$ is defined as follows

$$
B_{m, s} f(x)=\sum_{i=1}^{s}(-1)^{i+1}\left(\begin{array}{l}
s \\
i
\end{array}\right) B_{m}^{i} f(x) .
$$

Such GBs polynomials have been independently introduced and studied in [30], [17], [24].

By (3.19) and (3.18), for any $m, s \in \mathbb{N}$, the polynomial $B_{m, s} f$ takes the form

$$
B_{m, s} f(x)=\sum_{j=0}^{m} p_{m, j}^{(s)}(x) f\left(\frac{j}{m}\right), \quad 0 \leq x \leq 1,
$$

where

$$
p_{m, j}^{(s)}(x)=\sum_{i=1}^{s}\left(\begin{array}{l}
s \\
i
\end{array}\right)(-1)^{i-1} B_{m}^{i-1} p_{m, j}(x), \quad j=0, \ldots, m
$$

are the so-called fundamental Generalized Bernstein polynomials of degree $m$.

Note that the map $B_{m, s}: f \in C^{0}([0,1]) \rightarrow B_{m, s} f \in \mathbb{P}_{m}$ is a linear map, not always positive, and for $\forall m, s \in \mathbb{N}$, we have

$$
\begin{aligned}
& B_{m, s} f(0)=f(0) ; \quad B_{m, s} f(1)=f(1), \\
& B_{m, s} e_{i}(x)=x^{i}, i=1,2, \quad e_{i}(x):=x^{i} .
\end{aligned}
$$

For all degrees $m \in \mathbb{N}$, if we fix $s=1$, then we get the classical Bernstein polynomial, i.e., $B_{m, 1}=B_{m}$. For increasingly $s \in \mathbb{N}$, the sequence $\left\{B_{m, s} f\right\}_{s}$, continuously links Bernstein polynomials $B_{m} f$ to the Lagrange polynomials $L_{m} f \in \mathbb{P}_{m}$ interpolating $f$ at the nodes $\left\{t_{i}\right\}_{i=0}^{m}$, i.e.,

$$
L_{m} f(x)=\sum_{k=0}^{m} f\left(t_{k}\right) l_{m, k}(x), \quad l_{m, k}(x)=\prod_{k \neq i=0}^{m} \frac{x-t_{i}}{t_{k}-t_{i}} .
$$

Such property, advisable in different contexts (see [38], [34], [5]), was given in the following. 
Theorem 3.1. [24] For any $f \in C^{0}([0,1])$, we have

$$
\lim _{s \rightarrow \infty} B_{m, s} f(x)=L_{m} f(x)
$$

uniformly w.r.t. $x \in[0,1]$.

Figure 1 displays the behaviour of the fundamental GBs polynomials $p_{m, k}^{(s)}$, with fixed $m, k$ and increasing values of the parameter $s$. The plots confirm the continuous relation between Bernstein and Lagrange polynomials. In fact, as $s \rightarrow \infty$, in Figure 1 we see that the fundamental GBs polynomial $p_{m, k}^{(s)}(x)$ uniformly tends to the $k$-th fundamental Lagrange polynomial $l_{m, k}(x)$, according with Theorem 3.1.

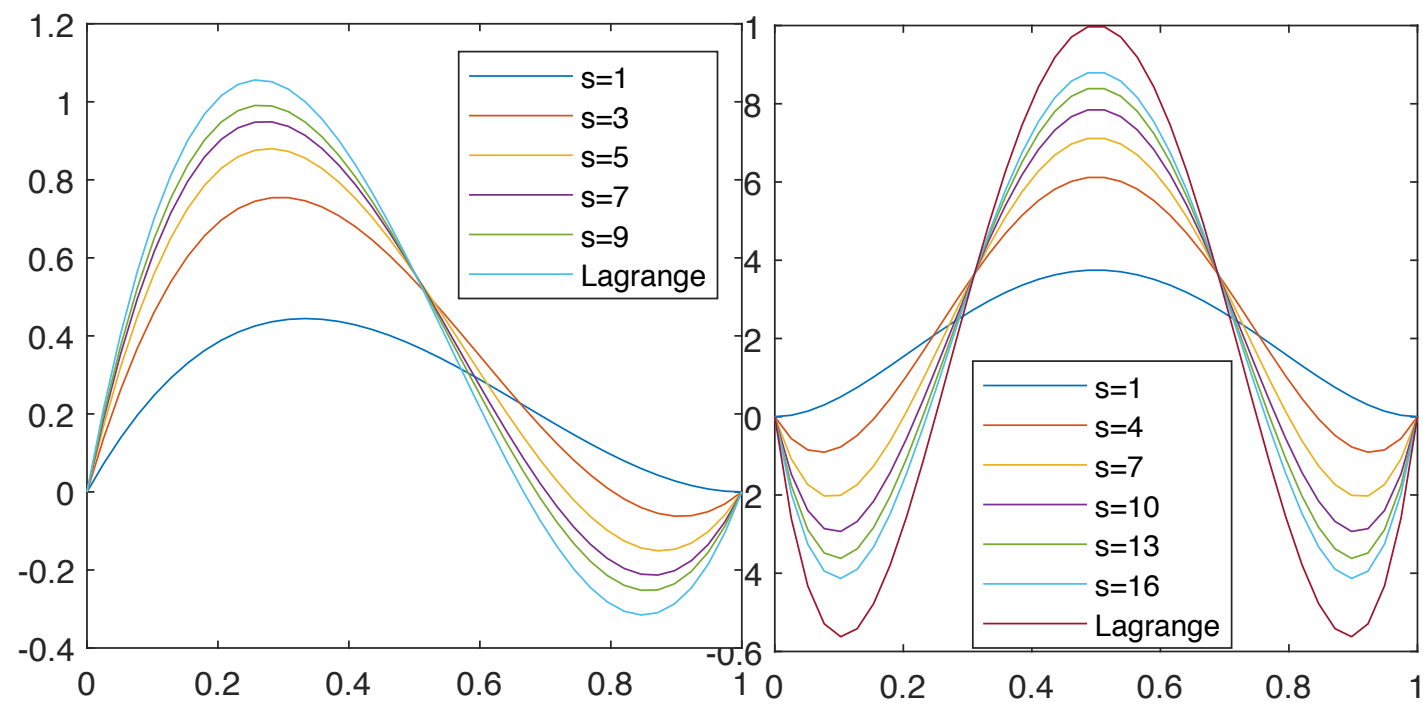

FIGURE 1. Plots of the fundamental GBs and Lagrange polynomials $p_{m, k}^{(s)}(x)$ and $l_{m, k}(x)$ for $m=3, k=1$ on the left and $m=2, k=2$ on the right

For all $m, s \in \mathbb{N}$, the fundamental GBs polynomials $\left\{p_{m, j}^{(s)}(x)\right\}_{j=0}^{m}$ form a partition of the unity, i.e.,

$$
\sum_{j=0}^{m} p_{m, j}^{(s)}(x)=1, \quad \forall x \in[0,1] .
$$
[34].

A handle vectorial form of the basis $\left\{p_{m, k}^{(s)}\right\}_{k=0}^{m}$ is given by the following theorem proved in

Theorem 3.2. For all $m, s \in \mathbb{N}$ and for any $x \in[0,1]$, let $\mathbf{p}_{m}^{(s)}(x)=\left[p_{m, 0}^{(s)}(x), p_{m, 1}^{(s)}(x), \ldots, p_{m, m}^{(s)}(x)\right]$ be the row-vector of fundamental GBs polynomials that, for $s=1$, reduces to the vector of fundamental Bernstein polynomials $\mathbf{p}_{m}(x)=\left[p_{m, 0}(x), \ldots, p_{m, m}(x)\right]$. Moreover, let $C_{m, s}$ be the following square matrix of order $(m+1)$

$$
\begin{aligned}
C_{m, s}: & =A^{-1}\left[I-(I-A)^{s}\right]=\left[I-(I-A)^{s}\right] A^{-1} \\
& =\left[I+(I-A)+(I-A)^{2}+\cdots+(I-A)^{s-1}\right],
\end{aligned}
$$


where $I$ is the identity matrix and $A \in \mathbb{R}^{(m+1) \times(m+1)}$ is defined by

$$
A_{i, j}=p_{m, j}\left(t_{i}\right), \quad t_{i}=\frac{i}{m}, \quad(i, j) \in N_{0}^{m} \times N_{0}^{m} .
$$

We have

$$
\mathbf{p}_{m}^{(s)}(x)=\mathbf{p}_{m}(x) \cdot C_{m, s}, \quad \forall m, s \in \mathbb{N}, \quad \forall x \in[0,1] .
$$

Moreover, for any $f \in C^{0}([0,1])$, the polynomial $B_{m, s} f$ can be represented in the following form

$$
B_{m, s} f(x)=\mathbf{p}_{m}(x) \cdot C_{m, s} \cdot \mathbf{f}_{m}, \quad \forall m, s \in \mathbb{N}, \quad \forall x \in[0,1],
$$

where $\mathbf{f}_{m} \in \mathbb{R}^{m+1}$ is the sampling (column) vector of the function $f$ evaluated at the nodes $t_{i}$, i.e.,

$$
\mathbf{f}_{m}:=\left[f(0), \ldots, f\left(\frac{i}{m}\right), \ldots, f(1)\right]^{T} .
$$

Remark 3.1. Theorem 3.2 provides a useful tool for computing GBs polynomial. Indeed from (3.27), it follows that the GBs polynomial $B_{m, s} f$ can be considered as the $m$-th classical Bernstein polynomial of a function $g$ having a suitable sampling vector, i.e.,

$$
B_{m, s} f(x)=B_{m} g(x)=\mathbf{p}_{m}(x) \cdot \mathbf{g}_{m},
$$

where the sampling vector of $g$ is given by

$$
\mathbf{g}_{m}:=C_{m, s} \cdot \mathbf{f}_{m} .
$$

As a consequence, we can compute the polynomial $B_{m, s} f$ by using the de Casteljau recursive scheme, which, as it is well-known, is a fast and stable algorithm [16].

Additional details on the fast computation of GBs polynomials are given in Section 7. In the sequel, we are going to analyze the approximation provided by GBs polynomials of fixed parameter $s \in \mathbb{N}$ and increasing degrees. About the estimate of the remainder term $f-B_{m, s} f$, the following error bound in $C^{0}([0,1])$ was proved in [19].

Theorem 3.3. [19] Let $s \in \mathbb{N}$ be fixed. Then, for all $m \in \mathbb{N}$ and any $f \in C^{0}([0,1])$, we have

$$
\left\|f-B_{m, s} f\right\| \leq \mathcal{C}\left\{\omega_{\varphi}^{2 s}\left(f, \frac{1}{\sqrt{m}}\right)+\frac{\|f\|}{m^{s}}\right\}, \quad C \neq \mathcal{C}(m, f) .
$$

Moreover, for any $0<\mu \leq 2 s$, we obtain

$$
\left\|f-B_{m, s} f\right\|=\mathcal{O}\left(m^{-\frac{\mu}{2}}\right), m \rightarrow \infty \Longleftrightarrow \omega_{\varphi}^{2 s}(f, t)=\mathcal{O}\left(t^{\mu}\right)
$$

and the 0 -saturation class is characterized by the equivalence

$$
\left\|f-B_{m, s} f\right\|=o\left(m^{-s}\right) \Longleftrightarrow f \text { is a linear function. }
$$

Applying the properties of the moduli of smoothness given in Section 2, several error estimates can be deduced from (3.29). In particular, for all $m, s \in \mathbb{N}$, by (2.11) and by (2.17), we have

$$
\begin{array}{lll}
\left\|f-B_{m, s} f\right\| \leq \frac{\mathcal{C}}{\sqrt{m^{r}}}, & \mathcal{C} \neq \mathcal{C}(m), & \forall f \in W_{r}, \quad r \leq 2 s, \\
\left\|f-B_{m, s} f\right\| \leq \frac{\mathcal{C}}{\sqrt{m^{\lambda}}}, & \mathcal{C} \neq \mathcal{C}(m), \quad \forall f \in Z_{\lambda}, \quad \lambda<2 s .
\end{array}
$$

Hence, we remark that by introducing the additional parameter $s \in \mathbb{N}$, the saturation order $m^{-1}$ occurring with classical Bernstein polynomials is enlarged to $\mathrm{m}^{-s}$ and, using the same function samples at the $(m+1)$ equidistant nodes of $[0,1]$, the GBs polynomial may provide 
the square root of the order of the best uniform polynomial approximation of $f$ in $\mathbb{P}_{m}$ (see (2.16)).

Nevertheless, $s$ cannot be chosen arbitrarily high and the reason is given by Theorem 3.1, stating that for $s \rightarrow \infty$ the operator $B_{m, s}$ tends to the Lagrange interpolating polynomial on equispaced points, which is a well known unstable operator.

In order to show the real degree in approximating a given function $f$, for increasing values of $m$ and $s$, in Table 1 , we report the maximum errors

$$
\mathcal{E}_{m, s} f=\max _{x \in \mathcal{X}}\left|f(x)-B_{m, s} f(x)\right|,
$$

attained in a sufficiently large set $\mathcal{X} \subset[0,1]$, for the test function $f(x)=|x-0.6|^{\frac{7}{2}} \in Z_{\frac{7}{2}}$, whose theoretical error goes like $\mathcal{O}\left(m^{-\frac{7}{4}}\right)$. In each column of Table 1 , the errors for $m$ fixed and $s$ varying, starting from $2^{6}$ on, until the errors decrease, are reported. The empty boxes mean that for the corresponding $s$, the error does not decrease anymore. We note that for any $m$ there

\begin{tabular}{|c|l|l|l|l|l|l|l|}
\hline$s$ & $m=16$ & $m=32$ & $m=64$ & $m=128$ & $m=256$ & $m=512$ & $m=1024$ \\
\hline $2^{6}$ & $2.50 e-5$ & $6.90 e-6$ & $1.95 e-6$ & $5.47 e-7$ & $1.49 e-7$ & $3.7 e-8$ & $9.86 e-9$ \\
\hline $2^{7}$ & $1.93 e-5$ & $5.20 e-6$ & $1.46 e-6$ & $4.10 e-7$ & $1.09 e-7$ & $2.76 e-8$ & $6.81 e-9$ \\
\hline $2^{8}$ & $3.78 e-5$ & $4.08 e-6$ & $1.15 e-6$ & $3.20 e-7$ & $8.45 e-8$ & $2.16 e-8$ & $4.58 e-9$ \\
\hline $2^{9}$ & & $3.31 e-6$ & $9.34 e-7$ & $2.57 e-7$ & $6.69 e-8$ & $1.73 e-8$ & $2.98 e-9$ \\
\hline $2^{10}$ & & & $7.34 e-7$ & $2.11 e-7$ & $5.42 e-8$ & $1.46 e-8$ & $2.07 e-9$ \\
\hline $2^{11}$ & & & $6.52 e-7$ & $1.77 e-7$ & $4.47 e-8$ & $1.22 e-8$ & $2.07 e-9$ \\
\hline $2^{12}$ & & & & $1.50 e-7$ & $3.74 e-8$ & $1.02 e-8$ & $2.04 e-9$ \\
\hline $2^{13}$ & & & & $1.29 e-7$ & $3.31 e-8$ & $8.44 e-9$ & $1.97 e-9$ \\
\hline $2^{14}$ & & & & $1.12 e-7$ & $2.93 e-8$ & $6.98 e-9$ & $1.89 e-9$ \\
\hline $2^{15}$ & & & & $9.89 e-8$ & $2.59 e-8$ & $5.74 e-9$ & $1.79 e-9$ \\
\hline $2^{16}$ & & & & & $2.29 e-8$ & $4.70 e-9$ & $1.67 e-9$ \\
\hline $2^{17}$ & & & & & $2.02 e-8$ & $3.81 e-9$ & $1.60 e-9$ \\
\hline $2^{18}$ & & & & & $1.79 e-8$ & $3.24 e-9$ & $1.50 e-9$ \\
\hline
\end{tabular}

TABLE 1. Convergence behaviour w.r.t. $m$ and $s$

exist a threshold $\widetilde{s}=\widetilde{s}(m)$, until which the errors decrease, while for $s>\widetilde{s}$ the situation is quite reversed. In these reverse cases, $m$ has to be increased for speeding up again the convergence. To highlight this behaviour, in Fig. 2, we plotted the error curves w.r.t. the same function $f(x)=|x-0.6|^{\frac{7}{2}}$, for fixed $m$ and $s$ varying from $2^{6}$ to $2^{18}$.

The investigation on the mutually relation between $m$ and $s$ for obtaining the optimal $s$ for each $m$ is still an open problem.

In Figure 3, the plots of the polynomials $B_{m, s} f$, for $m$ fixed and $s$ varying, are given. Since in the whole interval the curves seem to coincide, on the right a magnification is given in the restricted interval $[0.5,0.7]$.

In conclusion of the section, let us consider the case that $f$ is a continuously differentiable function up to a certain order $k \in \mathbb{N}$. In this case, GBs polynomials can be used for the simultaneous approximation of $f$ and its first $k$ derivatives. In fact, we have

$$
\lim _{m \rightarrow \infty}\left\|f^{(k)}-\left(B_{m, s} f\right)^{(k)}\right\|=0, \quad \forall f \in C^{k},
$$




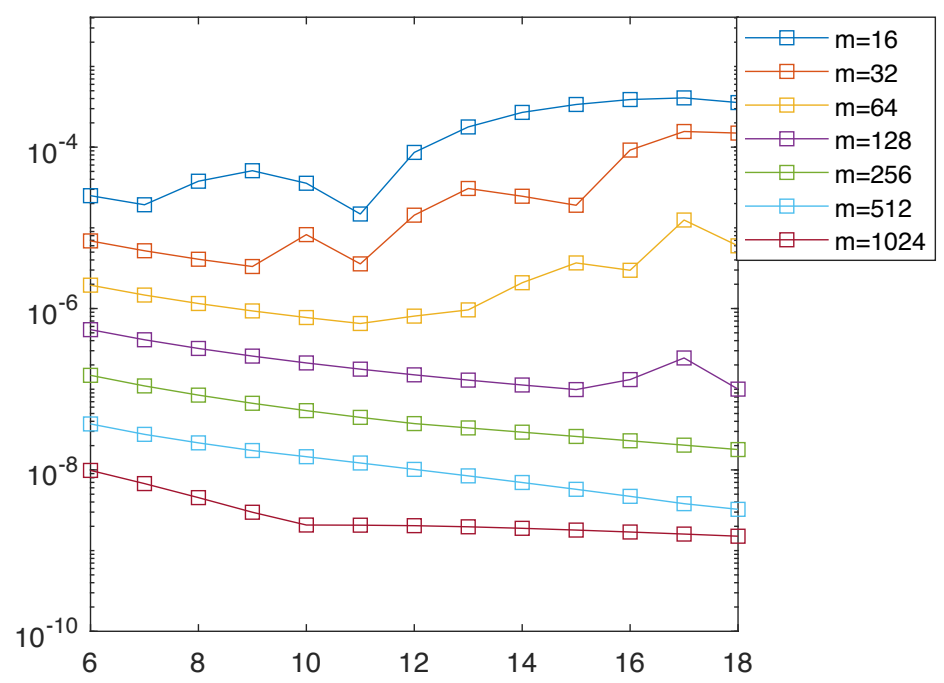

FIGURE 2. Plots of $\mathcal{E}_{m, s} f$ for $s=2^{n}, n=6,7, \ldots, 18$. On the abscissas the values of $n$ are reported.

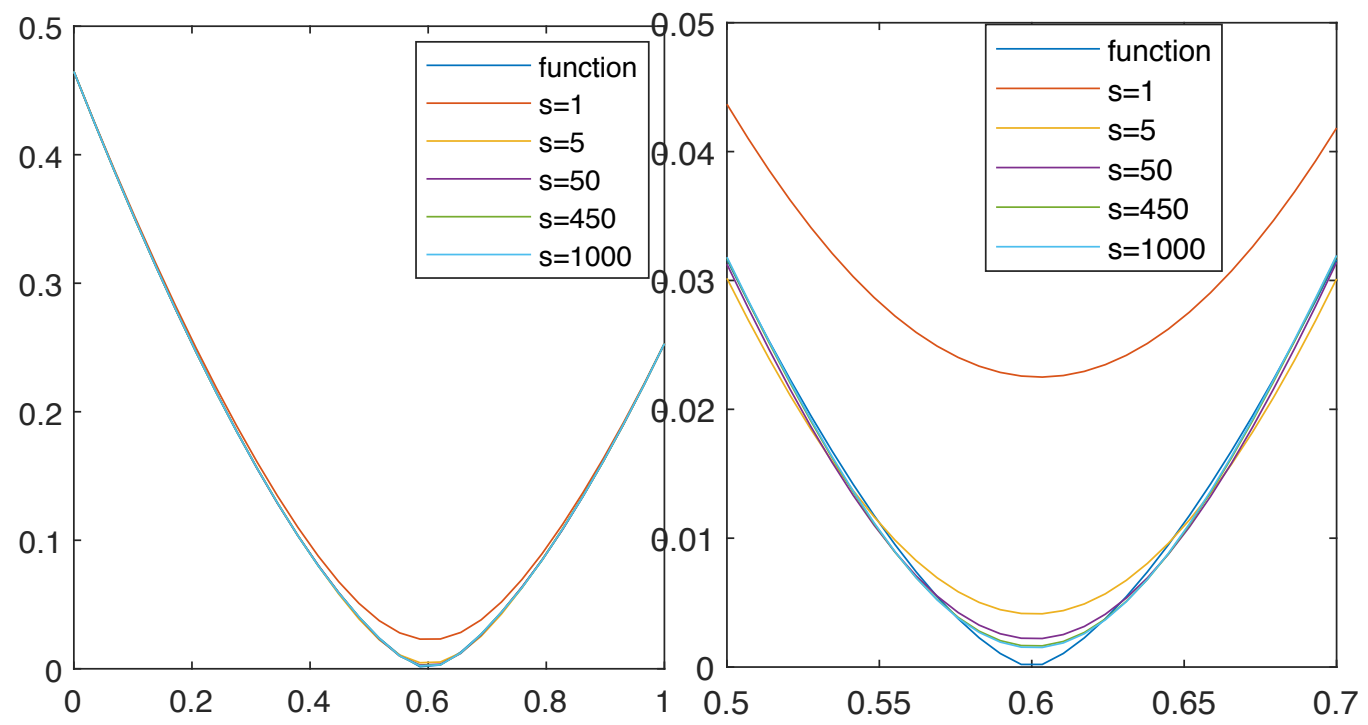

FIGURE 3. On the left the plots of the functions $f(x)=|x-0.6|^{\frac{7}{2}}$ and $B_{m, s} f$ for $m=31$ and different values of $s$. On the right the same plots are zoomed in $[0.5,0.7]$.

where the derivatives of GBs polynomials are all based on the same sampling vector of $f$. Some computational details on the derivatives of the GBs polynomials can be found in Section 7. Here, we recall the following error estimate. 
Theorem 3.4. [15, Corollary 1.6] Let $s \geq 1$ be fixed. Then, for all $m, k \in \mathbb{N}$ and any $f \in C^{k}$, we have

$$
\left\|\left(f-B_{m, s} f\right)^{(k)}\right\| \leq \mathcal{C}\left\{\begin{array}{ll}
\omega_{\varphi}^{2 s}\left(f^{\prime}, \frac{1}{\sqrt{m}}\right)+\omega^{s}\left(f^{\prime}, \frac{1}{m}\right)+\omega\left(f^{\prime}, \frac{1}{m^{s}}\right), & k=1 \\
\omega_{\varphi}^{2 s}\left(f^{(k)}, \frac{1}{\sqrt{m}}\right)+\omega^{s}\left(f^{(k)}, \frac{1}{m}\right)+\frac{\left\|f^{(k)}\right\|}{m^{s}}, & k \geq 2
\end{array},\right.
$$

where $\omega:=\omega^{1}$ and $\mathcal{C} \neq \mathcal{C}(m, f)$.

\section{A QUADRATURE RULE ON EQUAlLy SPACED KNOTS}

Based on GBs polynomials, the following quadrature rule was introduced in [24]

$$
\int_{0}^{1} f(x) d x=\Sigma_{m}^{(s)} f+R_{m}^{(s)} f
$$

where the quadrature sum is defined by

$$
\Sigma_{m}^{(s)} f:=\int_{0}^{1} B_{m, s} f(x) d x=\sum_{j=0}^{m} Q_{j}^{(s)} f\left(\frac{j}{m}\right), \quad Q_{j}^{(s)}:=\int_{0}^{1} p_{m, j}^{(s)}(x) d x
$$

and the quadrature error is given by

$$
R_{m}^{(s)} f=\int_{0}^{1}\left[f(x)-B_{m, s} f(x)\right] d x .
$$

Such rule is easy to construct since the quadrature weights are representable in the following form [35]

$$
Q_{j}^{(s)}=\frac{1}{m+1} \sum_{i=0}^{m}\left(C_{m, s}\right)_{i, j}, \quad j \in N_{0}^{m},
$$

where $\left(C_{m, s}\right)_{i, j}$ denotes the $(i, j)$-entry of the matrix $C_{m, s}$ in (3.24).

We point out that these quadrature weights are not always positive. Nevertheless, the quadrature formula is always stable. Indeed, by Theorem 3.3 and by the Uniform Boundedness Principle, it is possible to deduce the following theorem of convergence and stability.

Theorem 4.5. [32] For all $f \in C^{0}([0,1])$ and any $s, m \in \mathbb{N}$, there holds

$$
\left|R_{m}^{(s)} f\right| \leq \mathcal{C}\left(\omega_{\varphi}^{2 s}\left(f, \frac{1}{\sqrt{m}}\right)+\frac{\|f\|}{m^{s}}\right), \quad \mathcal{C} \neq \mathcal{C}(f, m), \quad \mathcal{C}=\mathcal{C}(s) .
$$

Moreover, the quadrature formula is stable, i.e.,

$$
\sup _{m} \sum_{j=0}^{m}\left|Q_{j}^{(s)}\right|<\infty .
$$

By estimate (4.34), it is possible to deduce the order of convergence for functions belonging to several functional spaces. For instance, if $f \in W_{r}$ with $r \in \mathbb{N}$, then for sufficiently large $m$ and for any integer $s \geq \frac{r}{2}$, we have by (2.10)

$$
\left|R_{m}^{(s)} f\right| \leq \mathcal{C}\left(\frac{\left\|f^{(r)} \varphi^{r}\right\|}{\sqrt{m^{r}}}+\frac{\|f\|}{m^{s}}\right), \quad \mathcal{C} \neq \mathcal{C}(f, m), \quad \mathcal{C}=\mathcal{C}(s) .
$$

Another example is given for $f \in Z_{\lambda}$. Indeed for any $s>\frac{\lambda}{2}$, by (2.17), we get

$$
\left|R_{m}^{(s)} f\right| \leq \mathcal{C} \frac{\|f\|_{Z_{\lambda}}}{\sqrt{m^{\lambda}}}, \quad \mathcal{C} \neq \mathcal{C}(f, m), \quad \mathcal{C}=\mathcal{C}(s) .
$$


Now, we propose two tests, comparing the performance of formula (4.33) with the classical Romberg integration scheme

$$
\int_{0}^{1} f(t) d t=T_{N, N} f+e_{N} f
$$

where $T_{N, N}$ is the Romberg rule and $e_{N} f$ denotes the quadrature error. This rule besides the well-known triangular scheme (see e.g. [11]), can be also represented as a linear combination of the samples of $f$

$$
T_{N, N} f=\sum_{i=0}^{2^{N}} \sigma_{i} f\left(\frac{i}{2^{N}}\right),
$$

that is a more convenient form in the implementation of other procedure, for instance in Nyström methods for integral equations. Details on the coefficients $\sigma_{i}$ are given in [32]. Here, we only recall the following result about the convergence which holds true for functions having a continuous derivative of fixed order $r \geq 1$ in [0,1] [7]:

$$
\left|e_{N} f\right| \leq \mathcal{C} \frac{2^{(r-1)^{2} / 4}}{(2 \pi)^{r}} \frac{\left\|f^{(r)}\right\|}{\left(2^{N}\right)^{r}}, \quad \forall N \geq \frac{r-1}{2}, \quad f \in C^{r}([0,1]),
$$

where $\mathcal{C}$ is a positive constant independent of $f$ and depending on $N$ and $r$ and such that $1.5 \leq \mathcal{C} \leq 3.1$.

\section{Example 4.1.}

$$
I\left(f_{1}\right)=\int_{0}^{1} \cos (x)(1-x)^{\frac{5}{2}} d x, \quad I\left(f_{2}\right)=\int_{0}^{1} \sqrt{1-\frac{\sin ^{2}(x)}{3}} d x .
$$

In order to compare rules (4.33) and (4.37), we choose $m=2^{N}$ in (4.33), reporting in Tables 2 and 3 the absolute errors. The empty boxes mean that no improvement is attained w.r.t. the errors obtained for the same values of $m$.

The values of the integrals $I\left(f_{1}\right) \sim 0.2744041660389273$ and $I\left(f_{2}\right) \sim 0.9526594143223039835$ were computed with 16 and 19 exact digits respectively by means of the software Mathematica.

\begin{tabular}{|c|l||l|l|l|}
\hline$m$ & Romberg & $s=32$ & $s=64$ & $s=2048$ \\
\hline 8 & $1.41 e-06$ & $1.35 e-06$ & $1.47 e-06$ & $9.04 e-07$ \\
\hline 16 & $1.47 e-07$ & $1.70 e-07$ & $1.39 e-07$ & $7.98 e-08$ \\
\hline 32 & $1.39 e-08$ & $1.53 e-08$ & $1.22 e-08$ & $6.51 e-09$ \\
\hline 64 & $1.28 e-09$ & $1.35 e-09$ & $1.07 e-09$ & $5.54 e-10$ \\
\hline 128 & $1.15 e-10$ & $1.19 e-10$ & $9.43 e-11$ & $4.81 e-11$ \\
\hline 256 & $1.03 e-11$ & $1.05 e-11$ & $8.31 e-12$ & $4.30 e-12$ \\
\hline 512 & $9.10 e-13$ & $9.31 e-13$ & $7.35 e-13$ & $3.91 e-13$ \\
\hline 1024 & $8.11 e-14$ & $8.26 e-14$ & $6.54 e-14$ & $1.67 e-14$ \\
\hline
\end{tabular}

TABLE 2. Errors for $I\left(f_{1}\right)$

Regarding $I\left(f_{1}\right)$, according to (4.35) and (4.38), since $f_{1} \in W_{5} \cap C^{2}([0,1])$ the error $R_{m}^{(s)} f$ will go as $\mathcal{O}\left(m^{-\frac{5}{2}}\right)$, while the error $e_{N}$ of the Romberg rule will behave like $\mathcal{O}\left(m^{-2}\right)$, where $m=2^{N}$.

About the second (elliptic) integral, we note that $f_{2} \in W_{r}$ for any $r$ and the convergence is very fast by both the quadrature rules. 


\begin{tabular}{|c|l||l|l|l|}
\hline$m$ & Romb & $s=64$ & $s=256$ & $s=1024$ \\
\hline 8 & $5.46 e-09$ & $5.33 e-09$ & $1.72 e-09$ & $4.44 e-11$ \\
\hline 16 & $2.29 e-11$ & $1.43 e-11$ & $6.26 e-13$ & $2.18 e-13$ \\
\hline 32 & $5.66 e-15$ & $1.33 e-15$ & $2.22 e-15$ & \\
\hline 64 & $1.11 e-16$ & $1.11 e-16$ & & \\
\hline 128 & $4.44 e-16$ & $1.11 e-15$ & & \\
\hline 256 & $2.22 e-16$ & $3.33 e-16$ & & \\
\hline
\end{tabular}

TABLE 3. Errors for $I\left(f_{2}\right)$

So, the tables show that the two quadrature rules are comparable for small values of $s$. However, in both the cases, by using the same number of samples $m$ the free parameter $s$ can be enhanced, allowing to gain better results.

We remark that in both the previous examples the speed of convergence is faster than the theoretical estimate of this speed. For instance, in the case of $I\left(f_{1}\right)$ with $m=1024$, the errors would be around $9.5 \times 10^{-7}$ for the Romberg formula and $2.8 \times 10^{-8}$ for the GBs rule. This means in particular that estimate (4.34) is not sharp.

So an open problem is to estimate $R_{m}^{(s)} f$ "directly" or, which is the same, to have an $L^{1}$ estimate of $f-B_{m, s} f$. In addition, it would be useful to understand how the constant $\mathcal{C}$ in (4.34) depends on $s$.

\section{A NYSTRÖM METHOD FOR SOLVING FREDHOLM INTEGRAL EQUATIONS}

Based on the quadrature rule (4.33), a Nyström method has been introduced in [32] for solving the following Fredholm Integral Equation (FIE)

$$
f(x)-\mu \int_{0}^{1} f(t) k(x, t) d t=g(x), \quad \mu \in \mathbb{R}, \quad x \in[0,1] .
$$

Such equation can be rewritten in operator form as follows

$$
(I-K) f=g,
$$

where $I$ denotes the identity operator and

$$
K f(x):=\mu \int_{0}^{1} k(x, t) f(t) d t, \quad x \in[0,1] .
$$

It is known [6] that if the kernel $k(x, t)$ is continuous, then $K: C^{0}([0,1]) \rightarrow C^{0}([0,1])$ is a compact operator.

In order to consider the case of more regular kernels, here and in the sequel, we will use the notation $k_{t}$ (respectively $k_{x}$ ) for the bivariate function $k(x, t)$ considered as a function of the single variable $x$ (respectively $t$ ). Using this notation, it is also known [20, Proposition 4.12] that if $k$ is continuous w.r.t. both the variables and we have

$$
\sup _{t \in[0,1]}\left\|k_{t}\right\|_{Z_{\lambda}}<+\infty, \quad \lambda>0,
$$

then $K: f \in C^{0}([0,1]) \rightarrow K f \in Z_{\lambda}$ is a countinuous map and hence, due to the compact embedding $Z_{\lambda} \subset C^{0}([0,1])$ ([20, Lemma3.2]), we have that $K: Z_{\lambda} \rightarrow Z_{\lambda}$ is a compact operator.

The previous mapping properties and the Fredholm Alternative yield the following theorem concerning the existence, the uniqueness and the degree of smoothness of the solution of the FIE (5.39). 
Theorem 5.6. Suppose that the homogeneous equation associated with (5.40) has only the trivial solution. If the kernel $k$ is continuous w.r.t. both the variables in $[0,1]$, then there exists a unique solution of (5.40), that is $f^{*} \in C^{0}([0,1])$, for any continuous function $g$.

If, in addition, for some $\lambda>0$ we have $k_{t} \in Z_{\lambda}$ uniformly w.r.t. $t \in[0,1]$, i.e., (5.42) holds, then (5.40) is uniquely solvable in the Hölder-Zygmund spaces $Z_{\rho}$ with $0<\rho \leq \lambda$, that is $f^{*} \in Z_{\rho}$, for all $g \in Z_{\rho}$.

In order to numerically solve the FIE (5.39), we recall that several fast convergent methods can be found in the literature concerning projection and Nyström methods based on GaussJacobi quadrature rules (see for instance [6] and [12] and the references therein).

Nevertheless, many problems in engineering and mathematical physics are often modelled by (5.39), where the only available data are discrete values of the kernel $k$ and the right-hand side $g$ at a uniform grid of nodes. In such cases, the implementation of all the methods based on Jacobi zeros needs a further approximation step in order to derive, from the available data, the sampling vectors at the involved Jacobi grid. On the other hand, classical methods based on piecewise polynomial approximation are also available (see [6]) but they offer lower degree of approximation.

On the contrary, the Nyström method based on the quadrature rule (4.33) can be directly applied to numerically solve the equation in the case that the values of the kernel $k$ and the term $g$ are known at equidistant nodes of $[0,1]$. To be more precise, in order to find a numerical approximation of the solution $f^{*}$, for all $m$, we consider the following approximation $K_{m}$ of the operator $K$

$$
K_{m} f(x)=\mu \sum_{i=0}^{m} Q_{i}^{(s)} k\left(x, t_{i}\right) f\left(t_{i}\right), \quad t_{i}=\frac{i}{m}, \quad s \in \mathbb{N},
$$

where $Q_{i}^{(s)}$ are the weights of the quadrature rule (4.33) that has been applied to $K f(x)$ given by (5.41). The operator $K_{m}$ defines the following approximate equation

$$
\left(I-K_{m}\right) f_{m}=g
$$

whose solution $f_{m}$, if existing, has to satisfy the following identity that is a consequence of (5.44) and (5.43)

$$
f_{m}(x)=\mu \sum_{i=0}^{m} Q_{i}^{(s)} k\left(x, t_{i}\right) \alpha_{i}^{*}+g(x), \quad \alpha_{i}^{*}:=f_{m}\left(t_{i}\right), \quad x \in[0,1] .
$$

This function is known as the Nyström interpolant of the solution $f^{*}$. In order to compute the unknowns $\alpha_{i}^{*}, i \in N_{0}^{m}$, we collocate the approximate equation (5.44) on the knots $t_{h}$, with $h \in N_{0}^{m}$, obtaining the following linear system of $(m+1)$ equations and $(m+1)$ unknowns $\left\{\alpha_{i}\right\}_{i \in N_{0}^{m}}$

$$
\alpha_{h}-\mu \sum_{i=0}^{m} Q_{i}^{(s)} k\left(t_{h}, t_{i}\right) \alpha_{i}=g\left(t_{h}\right), \quad t_{i}=\frac{i}{m}, \quad h \in N_{0}^{m} .
$$

The solution of this system, if existing, provides the values $\left\{\alpha_{i}^{*}\right\}_{i=0}^{m}$ that we need in (5.45) and vice versa, the values $f_{m}\left(t_{i}\right), i \in N_{0}^{m}$, are solutions of system (5.46). In other words, (5.44) and (5.46) are equivalent.

Denoting by $\mathbf{V}_{m}$ the coefficient matrix of the system (5.46) and by $\operatorname{cond}\left(\mathbf{V}_{m}\right)=\left\|\mathbf{V}_{m}\right\|_{\infty}$ $\left\|\mathbf{V}_{m}^{-1}\right\|_{\infty}$ its condition number w.r.t. the matrix infinity norm, we have the following result which extends a previous one stated for Sobolev spaces in [32]. 
Theorem 5.7. If for some $\lambda>0$, the kernel $k$ satisfies (5.42) and

$$
\sup _{x \in[0,1]}\left\|k_{x}\right\|_{Z_{\lambda}}<+\infty
$$

then for all integers $m \in \mathbb{N}$ and $s>\lambda / 2$, the system (5.46) is uniquely solvable and well-conditioned, i.e.,

$$
\operatorname{cond}\left(\mathbf{V}_{m}\right) \leq \mathcal{C}, \quad \mathcal{C} \neq \mathcal{C}(m)
$$

Moreover, for all $g \in Z_{\lambda}$ the unique solution $f^{*} \in Z_{\lambda}$ of the FIE (5.39) can be approximated by its Nyström interpolant $f_{m}$ in (5.45) according with the following error estimate

$$
\left\|f^{*}-f_{m}\right\| \leq \mathcal{C} \frac{\|f\|_{Z_{\lambda}}}{\sqrt{m^{\lambda}}},
$$

where $\mathcal{C} \neq \mathcal{C}\left(m, f^{*}\right)$ and $\mathcal{C}=\mathcal{C}(s)$.

Proof. The proof can be led using classical arguments (see for instance [6, Th.4.1.2]). Indeed, it is well known that if the Nyström method is based on a quadrature formula converging for continuous functions, then the linear system (5.46) in uniquely solvable and the condition number of the matrix of coefficients is bounded by $\left\|I-K_{m}\right\|\left\|\left(I-K_{m}\right)^{-1}\right\|$ that is uniformly bounded for the collectively compactness of the sequence $\left\{K_{m}\right\}_{m}$. Moreover, it is also known that

$$
\left\|f^{*}-f_{m}\right\| \sim\left\|K f^{*}-K_{m} f^{*}\right\| .
$$

Therefore, the Theorem is proved if we estimate the quadrature error for the function $k_{x} f^{*}$. Taking into account (4.36), we have just to estimate $\left\|f^{*} k_{x}\right\|_{Z_{\lambda}}$, being $f^{*}, k_{x} \in Z_{\lambda}$, uniformly w.r.t. $x$. Fix $x \in[0,1]$. Using (2.15), we have

$$
\left\|f^{*} k_{x}\right\|_{\lambda} \sim\left\|f^{*} k_{x}\right\|+\sup _{n}(n+1)^{\lambda} E_{n}\left(f^{*} k_{x}\right) .
$$

In the case $n=2 m$ is not hard to prove that

$$
E_{2 m}\left(f^{*} k_{x}\right) \leq 2\left\|k_{x}\right\| E_{m}\left(f^{*}\right)+\left\|f^{*}\right\| E_{m}\left(k_{x}\right) .
$$

Analogously if $n=2 m+1$, then

$$
E_{2 m+1}\left(f^{*} k_{x}\right) \leq 2\left\|k_{x}\right\| E_{m+1}\left(f^{*}\right)+\left\|f^{*}\right\| E_{m}\left(k_{x}\right) .
$$

Therefore,

$$
\left\|f^{*} k_{x}\right\|+\sup _{n}(n+1)^{\lambda} E_{n}\left(f^{*} k_{x}\right) \leq \mathcal{C}\left(\left\|k_{x}\right\|\left\|f^{*}\right\|_{Z_{\lambda}}+\left\|f^{*}\right\|\left\|k_{x}\right\|_{Z_{\lambda}}\right) .
$$

Hence, assuming the sup on $x \in[0,1]$ and holding (5.47), we finally get

$$
\sup _{x}\left\|f^{*} k_{x}\right\|_{Z_{\lambda}} \leq \mathcal{C}\left\|f^{*}\right\|_{Z_{\lambda}}, \quad \mathcal{C} \neq \mathcal{C}\left(m, f^{*}\right)
$$

and (5.48) follows.

Remark 5.2. The convergence estimate (5.48) says that if the known functions in equation (5.39) are in $Z_{\lambda}$, then the order of convergence is $\mathcal{O}\left(m^{-\frac{\lambda}{2}}\right)$. This means that in the Hölder-Zygmund spaces the method converges with an order that is the half of the order of the best polynomial approximation in $Z_{\lambda}$ (see (2.16)).

In the sequel, we propose a numerical test in order to check the previous theoretical estimate. 
Example 5.2. We consider the following equation

$$
f(x)-0.2 \int_{0}^{1} f(t)|x-t|^{7.5} d t=|\arctan (x-0.5)|^{10.4} .
$$

Here, $\mu=0.2$, the kernel $k(x, t)=|x-t|^{7.5} \in Z_{7.5}$ w.r.t. both the variables and $g(x)=\mid \arctan (x-$ $0.5)\left.\right|^{10.4} \in Z_{10.4}$. In Table 4, we report the maximum errors attained in a discrete sufficiently large set of point in $[0,1]$, for increasing values of $m$ and $s$.

\begin{tabular}{|c|l|l|l|}
\hline$m$ & $s=16$ & $s=32$ & $s=64$ \\
\hline 16 & $0.71 e-02$ & $0.34 e-02$ & $0.16 e-02$ \\
\hline 32 & $0.58 e-04$ & $0.43 e-05$ & $0.37 e-06$ \\
\hline 64 & $0.41 e-07$ & $0.15 e-09$ & $0.15 e-10$ \\
\hline 128 & $0.18 e-11$ & $0.27 e-14$ & $0.24 e-14$ \\
\hline 256 & $0.12 e-14$ & $\mathbf{0 . 3 3 e}-\mathbf{1 5}$ & $\mathbf{0 . 6 9 e}-\mathbf{1 5}$ \\
\hline
\end{tabular}

TABLE 4. Errors by means of the Nyström interpolant

According with (5.48) the theoretical error behaves like $\mathcal{O}\left(\mathrm{m}^{-15 / 4}\right)$ for $s>3$ and as shown in Table 4, the Nyström method goes faster than the attended speed of convergence. For instance, the machine precision is attained for $m=256, s=32$.

\section{SimultaneOUs APPROXIMATION OF THE HilbERT TRANSFORM AND ITS FIRST DERIVATIVE}

For any $f \in C^{0}([0,1])$, let

$$
\mathcal{H} f(t)=f_{0}^{1} \frac{f(x)}{x-t} d x \quad \text { and } \quad \mathcal{H}^{1} f(t)=f_{0}^{1} \frac{f(x)}{(x-t)^{2}} d x, \quad 0<t<1,
$$

be the (finite) Hilbert and Hadamard transforms of $f$, respectively, where we used the single and double bar-integral notation to indicate that the integrals have to be understood as the Cauchy principal value integral and the Hadamard finite-part integral, respectively, namely (see for instance [31], [37, (1.3)])

$$
\begin{gathered}
\mathcal{H} f(t)=\lim _{\epsilon \rightarrow 0}\left[\int_{0}^{t-\epsilon} \frac{f(x)}{x-t} d x+\int_{t+\epsilon}^{1} \frac{f(x)}{x-t} d x\right], \quad 0<t<1, \\
\mathcal{H}^{1} f(t)=\lim _{\varepsilon \rightarrow 0}\left[\int_{0}^{t-\varepsilon} \frac{f(x)}{(x-t)^{2}} d x+\int_{t+\varepsilon}^{1} \frac{f(x)}{(x-t)^{2}} d x-\frac{2 f(t)}{\varepsilon}\right], \quad 0<t<1 .
\end{gathered}
$$

An alternative definition interprets the Hadamard transform as the first derivative of the Hilbert transform, i.e.,

$$
\mathcal{H}^{1} f(t)=\frac{d}{d t} \int_{0}^{1} \frac{f(x)}{x-t} d x, \quad 0<t<1
$$

and in that case that $f^{\prime}$ is Hölder continuous on $[0,1]$, the definitions (6.51) and (6.50) are indeed equivalent (see [37]).

Both the previous transforms are widely used in many areas of mathematical physics (potential theory, fracture mechanics, aerodynamics, elasticity, etc.), where several boundary-value problems can be formulated as singular integral equations in $[0,1]$ involving such integrals (see e.g. $[21,27,28,29,37]$ and the references therein).

The following theorem provide some upper bounds of $|\mathcal{H} f(t)|$ and $\left|\mathcal{H}^{1} f(t)\right|$, respectively, in the case that a Dini-type condition is satisfied by $f$ and $f^{\prime}$, respectively. We omit the proof since 
it can be deduced mutatis mutandis by the analogous results in [9, Th.2.1],[18], concerning the case $[-1,1]$.

Theorem 6.8. Let $0<t<1$. For all functions $f$ such that

$$
\int_{0}^{1} \frac{\omega_{\varphi}(f, u)}{u} d u<\infty
$$

we have

$$
\log ^{-1}\left(\frac{e}{t(1-t)}\right)|\mathcal{H} f(t)| \leq \mathcal{C}\left(\|f\|+\int_{0}^{1} \frac{\omega_{\varphi}(f, u)}{u} d u\right), \quad \mathcal{C} \neq \mathcal{C}(f, t) .
$$

Moreover, if

$$
\int_{0}^{1} \frac{\omega_{\varphi}\left(f^{\prime}, u\right)}{u} d u<\infty
$$

then we have

$$
\varphi^{2}(t)\left|\mathcal{H}^{1} f(t)\right| \leq \mathcal{C}\left(\|f\|+\int_{0}^{1} \frac{\omega_{\varphi}\left(f^{\prime}, \tau\right)}{\tau} d \tau\right), \quad \mathcal{C} \neq \mathcal{C}(f, t) .
$$

In many applications, the data are discrete and often consist of only the values of $f$ at equidistant points of $[0,1]$. In this case, the simultaneous approximation properties of GBs polynomials turn out to be useful in constructing quadrature rules for the simultaneous approximation of the Hilbert and Hadamard transforms.

As regards the Hilbert transform, a first numerical approach based on GBs polynomials can be found in $[25,26]$. Such method has been recently improved in [18], where efficient quadrature rules provide the simultaneous approximation of $\mathcal{H} f(t)$ and $\mathcal{H}^{1} f(t)$ by using the same samples of $f$, taken at equidistant nodes of $[-1,1]$. Here, we consider similar quadrature rules on the interval $[0,1]$.

Such formulas have been constructed starting from the following standard decompositions

$$
\begin{aligned}
\mathcal{H} f(t) & =\int_{0}^{1} \frac{f(x)-f(t)}{x-t} d x+f(t) \log \left(\frac{1-t}{t}\right) \\
\mathcal{H}^{1} f(t) & =\int_{0}^{1} \frac{f(x)-f(t)-f^{\prime}(t)(x-t)}{(x-t)^{2}} d x+f^{\prime}(t) \log \left(\frac{1-t}{t}\right)-\frac{f(t)}{t(1-t)}
\end{aligned}
$$

that are based on the ordinary integrals

$$
\begin{aligned}
\mathcal{F} f(t) & :=\int_{0}^{1} \frac{f(x)-f(t)}{x-t} d x, \\
\mathcal{F}^{1} f(t) & :=\int_{0}^{1} \frac{f(x)-f(t)-f^{\prime}(t)(x-t)}{(x-t)^{2}} d x .
\end{aligned}
$$

These integrals are approximated by the following quadrature rules based on the GBs polynomials

$$
\begin{aligned}
\mathcal{F} f(t) & =\mathcal{F}_{m, s} f(t)+\Phi_{m, s} f(t) \\
\mathcal{F}^{1} f(t) & =\mathcal{F}_{m, s}^{1} f(t)+\Phi_{m, s}^{1} f(t),
\end{aligned}
$$


where $\Phi_{m, s} f$ and $\Phi_{m, s}^{1} f$ denotes the quadrature errors and $\mathcal{F}_{m, s} f, \mathcal{F}_{m, s}^{1} f$ are quadrature sums defined as follows

$$
\begin{aligned}
\mathcal{F}_{m, s} f(t) & :=\int_{0}^{1} \frac{B_{m, s} f(x)-B_{m, s} f(t)}{x-t} d x \\
\mathcal{F}_{m, s}^{1} f(t) & :=\int_{0}^{1} \frac{B_{m, s} f(x)-B_{m, s} f(t)-\left(B_{m, s} f\right)^{\prime}(t)(x-t)}{(x-t)^{2}} d x .
\end{aligned}
$$

Recalling (3.20), the above integrals can be written as follows

$$
\begin{array}{ll}
\mathcal{F}_{m, s} f(t)=\sum_{j=0}^{m} f\left(\frac{j}{m}\right) D_{m, j}^{(s)}(t), & D_{m, j}^{(s)}(t):=\int_{0}^{1} \frac{p_{m, j}^{(s)}(x)-p_{m, j}^{(s)}(t)}{x-t} d x \\
\mathcal{F}_{m, s}^{1} f(t)=\sum_{j=0}^{m} f\left(\frac{j}{m}\right) \bar{D}_{m, j}^{(s)}(t), & \bar{D}_{m, j}^{(s)}(t):=\frac{d}{d t} D_{m, j}^{(s)}(t) .
\end{array}
$$

Thus, assuming at first instance that the values $f(t), f^{\prime}(t)$ are available, we get the following quadrature rules for the Hilbert and Hadamard transforms at the point $t \in(0,1)$

$$
\begin{aligned}
\mathcal{H} f(t) & =\mathcal{F}_{m, s} f(t)+f(t) \log \left(\frac{1-t}{t}\right)+\Phi_{m, s} f(t) \\
& =: H_{m, s} f(t)+\Phi_{m, s} f(t),
\end{aligned}
$$

$$
\begin{aligned}
\mathcal{H}^{1} f(t) & =\mathcal{F}_{m, s}^{1} f(t)+f^{\prime}(t) \log \left(\frac{1-t}{t}\right)-\frac{f(t)}{t(1-t)}+\Phi_{m, s}^{1} f(t) \\
& =: H_{m, s}^{1} f(t)+\Phi_{m, s}^{1} f(t) .
\end{aligned}
$$

In the case that the values of $f(t)$ and $f^{\prime}(t)$ are unknown, we approximate them by $B_{m, s} f(t)$ and its derivative, respectively, obtaining

$$
\begin{aligned}
\mathcal{H} f(t) & =\mathcal{F}_{m, s} f(t)+\log \left(\frac{1-t}{t}\right) B_{m, s} f(t)+\mathcal{E}_{m, s} f(t) \\
& =: \mathcal{H}_{m, s} f(t)+\mathcal{E}_{m, s} f(t)
\end{aligned}
$$

and

$$
\begin{aligned}
\mathcal{H}^{1} f(t) & =\mathcal{F}_{m, s}^{1} f(t)+\log \left(\frac{1-t}{t}\right)\left(B_{m, s} f\right)^{\prime}(t)-\frac{1}{t(1-t)} B_{m, s} f(t)+\mathcal{E}_{m, s}^{1} f(t) \\
& =: \mathcal{H}_{m, s}^{1} f(t)+\mathcal{E}_{m, s}^{1} f(t),
\end{aligned}
$$

where $\mathcal{E}_{m, s} f(t), \mathcal{E}_{m, s}^{1} f(t)$ denote the errors and the remaining part at the right-hand side of (6.63), (6.64) reduces to a quadrature sum based on the same samples of $f$.

Further numerical details on the computation of the previous quadrature rules will be given in Section 7. In the sequel, we are going to discuss the convergence rate of such formulas.

The following result has been stated in [18] for the interval $[-1,1]$.

Theorem 6.9. Let $0<t<1$. For all $f \in C^{0}([0,1])$ satisfying (6.52), we get

$$
\left|\mathcal{E}_{m, s} f(t)\right| \leq \mathcal{C} \log \left(\frac{e}{t(1-t)}\right)\left[\log m\left\|f-B_{m, s} f\right\|+\int_{0}^{\frac{1}{m}} \frac{\omega_{\varphi}^{r}(f, u)}{u} d u\right], \quad \mathcal{C} \neq \mathcal{C}(m, f, t) .
$$

Moreover, in the case that (6.53) holds, we have

$$
\varphi^{2}(t)\left|\mathcal{E}_{m, s}^{1} f(t)\right| \leq \mathcal{C}\left[\left\|f-B_{m, s} f\right\|+\log m\left\|\left(f-B_{m, s} f\right)^{\prime}\right\|+\int_{0}^{\frac{1}{m}} \frac{\omega_{\varphi}^{r}\left(f^{\prime}, u\right)}{u} d u\right]
$$


with $r<m$ and $\mathcal{C} \neq \mathcal{C}(m, f, t)$.

Finally, the errors $\Phi_{m, s} f(t)$ and $\Phi_{m, s}^{1} f(t)$ satisfy the same estimates of $\mathcal{E}_{m, s} f(t)$ and $\mathcal{E}_{m, s}^{1} f(t)$, and in addition we have

$$
\begin{aligned}
\left|\Phi_{m, s} f(t)\right| \leq \mathcal{C}\left\|\left(f-B_{m, s} f\right)^{\prime}\right\|, & \forall f \in C^{1}([0,1]), & \mathcal{C} \neq \mathcal{C}(m, f, t), \\
\left|\Phi_{m, s}^{1} f(t)\right| \leq \mathcal{C}\left\|\left(f-B_{m, s} f\right)^{\prime \prime}\right\|, & \forall f \in C^{2}([0,1]), & \mathcal{C} \neq \mathcal{C}(m, f, t) .
\end{aligned}
$$

From this theorem, several error estimates of the quadrature errors can be obtained by the error estimates of GBs polynomials recalled in Section 3 and based on several moduli of smoothness of $f$ and $f^{\prime}$. For instance, as regards the approximation of the Hilbert transform, by (3.29) and Theorem 3.4, we get that

$$
\log ^{-1}\left(\frac{e}{t(1-t)}\right)\left|\mathcal{E}_{m, s} f(t)\right| \leq \mathcal{C} \log m\left[\omega_{\varphi}^{2 s}\left(f, \frac{1}{\sqrt{m}}\right)+\frac{\|f\|}{m^{s}}\right]+\mathcal{C} \int_{0}^{\frac{1}{m}} \frac{\omega_{\varphi}^{r}(f, u)}{u} d u
$$

holds with $r<m$ and $\mathcal{C} \neq \mathcal{C}(m, f, t)$, for any $f$ satisfying (6.52). Moreover,

$$
\left|\Phi_{m, s} f(t)\right| \leq \mathcal{C}\left[\omega_{\varphi}^{2 s}\left(f^{\prime}, \frac{1}{\sqrt{m}}\right)+\omega^{s}\left(f^{\prime}, \frac{1}{m}\right)+\omega\left(f^{\prime}, \frac{1}{m^{s}}\right)\right], \quad \forall f \in C^{1}([0,1]) .
$$

For brevity, we omit the details and only state the following corollary to Theorem 6.9, which easily follows by using (2.10) and (2.17) in the estimates of Theorem 3.3 and Theorem 3.4.

Corollary 6.1. Let $0<t<1$. For any given $s \in \mathbb{N}$ and sufficiently large $m \in \mathbb{N}$, as regards the quadrature errors for the Hilbert transform, we have

$$
\begin{aligned}
&\left|\mathcal{E}_{m, s} f(t)\right| \leq \mathcal{C} \log \left(\frac{e}{t(1-t)}\right) \frac{\|f\|_{W_{r}}}{\sqrt{m^{r}}} \log m, \forall f \in W_{r}, \quad r \leq 2 s, \\
&\left|\mathcal{E}_{m, s} f(t)\right| \leq \mathcal{C} \log \left(\frac{e}{t(1-t)}\right) \frac{\|f\|_{Z_{\lambda}}}{\sqrt{m^{\lambda}}} \log m, \quad \forall f \in Z_{\lambda}, \quad 0<\lambda<2 s,
\end{aligned}
$$

with $\mathcal{C} \neq \mathcal{C}(m, f, t)$. The same estimate holds for $\left|\Phi_{m, s} f(t)\right|$, which also satisfies

$$
\left|\Phi_{m, s} f(t)\right| \leq \frac{\mathcal{C}}{\sqrt{m^{k}}}, \quad \forall f \in C^{k+1}([0,1]), 1 \leq k \leq 2 s, \quad \mathcal{C} \neq \mathcal{C}(m, t) .
$$

Moreover, concerning the approximation of the Hadamard transform, we have

$$
\varphi^{2}(t)\left|\mathcal{E}_{m, s}^{1} f(t)\right| \leq \mathcal{C} \frac{\log m}{\sqrt{m^{k}}}, \quad \forall f \in C^{k+1}([0,1]), 1 \leq k \leq 2 s, \quad \mathcal{C} \neq \mathcal{C}(m, t)
$$

and the error $\Phi_{m, s}^{1} f(t)$ satisfies the same estimate and

$$
\left|\Phi_{m, s}^{1} f(t)\right| \leq \frac{\mathcal{C}}{\sqrt{m^{k}}}, \quad \forall f \in C^{k+2}([0,1]), 1 \leq k \leq 2 s, \quad \mathcal{C} \neq \mathcal{C}(m, t) .
$$

We conclude by proposing the following test.

Example 6.3. Consider $\mathcal{H} f(t), \mathcal{H}^{1} f(t)$ with $f(x)=\frac{e^{x}}{1+x^{2}}$. By the previous algorithms, the reconstruction of $\mathcal{H} f(t)$ and $\mathcal{H}^{1} f(t)$, has been performed in high precision (more than 10 exact digits) by using only 200 samples of $f$ for both of them. In the graph are shown the approximation $H_{m, s} f(t)$ and $H_{m, s}^{1} f(t)$ of the functions $\mathcal{H} f(t)$ and $\mathcal{H}^{1} f(t)$, respectively. 


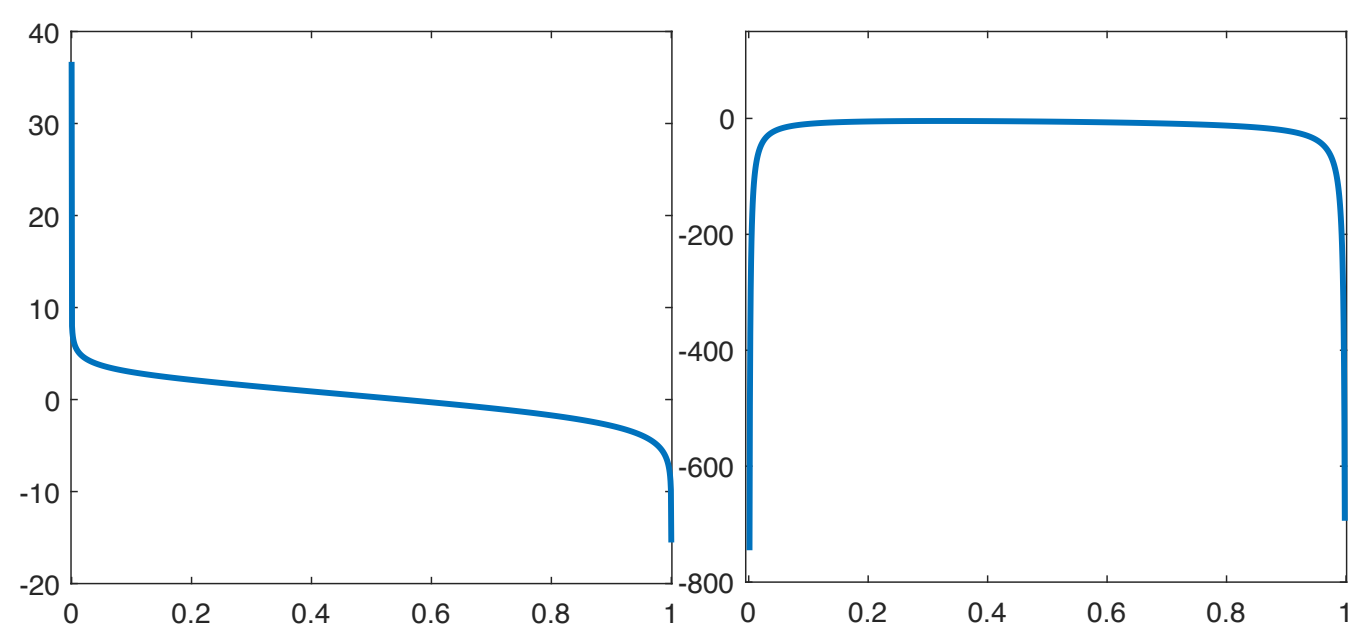

FIGURE 4. $H_{m, s} f$ and $H_{m, s}^{1} f, m=200, s=20$

\section{BIVARIATE GBS POLYNOMIALS AND APPLICATIONS}

7.1. The bivariate generalized Bernstein operator. Let $\mathrm{S}=[0,1]^{2}$ and $C(\mathrm{~S})$ indicate the space of continuous functions $f$ in two variables, equipped with the uniform norm on the square $\mathrm{S}$

$$
\|f\|_{\mathrm{S}}=\max _{(x, y) \in \mathrm{S}}|f(x, y)| .
$$

Denote by $\mathbf{m}=\left(m_{1}, m_{2}\right), m_{1}, m_{2} \in \mathbb{N}$ and by $\mathbf{s}=\left(s_{1}, s_{2}\right), s_{1}, s_{2} \in \mathbb{N}$. From now on, let $\mathbf{P}_{i j}=\left(\frac{i}{m_{1}}, \frac{j}{m_{2}}\right),(i, j) \in N_{0}^{m_{1}} \times N_{0}^{m_{2}}$ and $\mathbb{P}_{m_{1}, m_{2}}$ denote the space of the bivariate algebraic polynomials of degree $m_{1}$ w.r.t. the variable $x$ and $m_{2}$ w.r.t. the variable $y$.

With these notation and by $B_{m, s}$ given in (3.20), we can introduce the bivariate Generalized Bernstein operator $\mathbf{B}_{\mathrm{m}, \mathrm{s}}$ on $\mathrm{S}$ as the tensor product

$$
\mathbf{B}_{\mathbf{m}, \mathbf{s}}:=B_{m_{1}, s_{1}} \otimes B_{m_{2}, s_{2}}: C(\mathrm{~S}) \rightarrow \mathbb{P}_{m_{1}, m_{2}}, \quad \mathbf{m}=\left(m_{1}, m_{2}\right), \quad \mathbf{s}=\left(s_{1}, s_{2}\right) .
$$

This operator with $m_{1}=m_{2}$ and $s_{1}=s_{2}$ was introduced in [33]. Here, we are proposing a more general definition in order to get a more flexible approximation tool, according to the different smoothness properties of the approximating bivariate function, with respect to the single variables. In other words, we want to make the most of the advantages of the definition of $\mathbf{B}_{\mathbf{m}, \mathbf{s}}$ as a tensor product.

By definition and taking into account (3.20), the polynomial $\mathbf{B}_{\mathbf{m}, \mathbf{s}} f(x, y)$ can be expressed as

$$
\mathbf{B}_{\mathbf{m}, \mathbf{s}} f(x, y)=\sum_{i=0}^{m_{1}} \sum_{j=0}^{m_{2}} p_{m_{1}, i}^{\left(s_{1}\right)}(x) p_{m_{2}, j}^{\left(s_{2}\right)}(y) f\left(\mathbf{P}_{i j}\right)
$$

with $\left\{p_{m, k}^{(s)}\right\}_{k \in N_{0}^{m}}$ defined in (3.21). 
Let $f \in C(\mathrm{~S})$. For $\mathbf{s}=(1,1), \mathbf{B}_{\mathbf{m}, \mathbf{s}} f$ reduces to the bivariate Bernstein polynomial (see for instance [10] for the case $\left.m_{1} \equiv m_{2}\right)$,

$$
\mathbf{B}_{\mathbf{m}} f(x, y)=\sum_{i=0}^{m_{1}} \sum_{j=0}^{m_{2}} p_{m_{1}, i}(x) p_{m_{2}, j}(y) f\left(\mathbf{P}_{i j}\right) .
$$

Using the vector representation for the Bernstein basis, the bivariate Bernstein polynomial can be rewritten as

$$
\mathbf{B}_{\mathbf{m}} f(x, y)=\mathbf{p}_{m_{1}}(x)^{T} \mathbf{F}_{\mathbf{m}} \mathbf{p}_{m_{2}}(y),
$$

where the entries of the matrix $\mathbf{F}_{\mathbf{m}} \in \mathbb{R}^{\left(m_{1}+1\right) \times\left(m_{2}+1\right)}$ are

$$
\left(\mathbf{F}_{\mathbf{m}}\right)_{i, j}=f\left(\mathbf{P}_{i j}\right), \quad(i, j) \in N_{0}^{m_{1}} \times N_{0}^{m_{2}} .
$$

Extending some properties holding true in the univariate case, it is not hard to prove that $\mathbf{B}_{\mathbf{m}, \mathbf{s}} f$ interpolates $f$ at the corners of the square $\mathrm{S}$ and preserves bivariate polynomials of degree 1 in each variable separately.

By (3.26), $\mathbf{B}_{\mathbf{m}, \mathbf{s}} f$ can be also represented in the Bernstein basis. Indeed, using the definiton of $C_{m, s}$ given in (3.24), it results

$$
\mathbf{B}_{\mathbf{m}, \mathbf{s}} f(x, y)=\mathbf{p}_{m_{1}}(x)^{T} C_{m_{1}, s_{1}} \mathbf{F}_{\mathbf{m}} C_{m_{2}, s_{2}}^{T} \mathbf{p}_{m_{2}}(y) .
$$

Setting

$$
G_{\mathbf{m}, \mathbf{s}}=C_{m_{1}, s_{1}} \mathbf{F}_{\mathbf{m}} C_{m_{2}, s_{2}}^{T}
$$

by $(7.71)$, it follows

$$
\mathbf{B}_{\mathbf{m}, \mathbf{s}} f(x, y)=\mathbf{p}_{m_{1}}(x)^{T} G_{\mathbf{m}, \mathbf{s}} \mathbf{p}_{m_{2}}(y),
$$

i.e., according to (7.69) the polynomial $\mathbf{B}_{\mathbf{m}, \mathbf{s}} f$ can be seen as the bivariate Bernstein polynomial of a continuous function $g$ such that $g\left(\mathbf{P}_{i j}\right)=\left(G_{\mathbf{m}, \mathbf{s}}\right)_{i, j},(i, j) \in N_{0}^{m_{1}} \times N_{0}^{m_{2}}$.

From now on, let $f_{x}$ and $f_{y}$ denote the function $f(x, y)$ when considered as a function of the only variable $y$ and $x$, respectively.

We give a convergence result of the proposed approximation which is a generalization of that given in [33].

Theorem 7.10. Let $f \in C(\mathrm{~S})$. For any fixed $\mathbf{s}=\left(s_{1}, s_{2}\right)$, it results

$$
\left\|\mathbf{B}_{\mathbf{m}, \mathbf{s}} f\right\|_{\mathrm{S}} \leq 2^{s_{1}+s_{2}}\|f\|_{\mathrm{S}}, \quad \forall \mathbf{m} .
$$

Moreover, for $m_{1}$ and $m_{2}$ sufficiently large (say $m_{1}, m_{2}>m_{0}$ fixed)

$$
\begin{aligned}
\left\|f-\mathbf{B}_{\mathbf{m}, \mathbf{s}} f\right\|_{\mathrm{S}} & \leq \mathcal{C}\left\{\sup _{y \in[0,1]}\left[\omega_{\varphi}^{2 s_{1}}\left(f_{y}, \frac{1}{\sqrt{m_{1}}}\right)+\frac{\left\|f_{y}\right\|}{m_{1}^{s_{1}}}\right]\right. \\
& \left.+\sup _{x \in[0,1]}\left[\omega_{\varphi}^{2 s_{2}}\left(f_{x}, \frac{1}{\sqrt{m_{2}}}\right)+\frac{\left\|f_{x}\right\|}{m_{2}^{s_{2}}}\right]\right\},
\end{aligned}
$$

where $\mathcal{C}$ is a positive constant depending on $s_{1}, s_{2}$ and independent of $f, m_{1}$ and $m_{2}$.

Proof. The proof can be led repeating word by word that of Theorem 3.1 in [33]. Indeed, denoting by $\|A\|_{\infty}$, the infinity norm of a matrix $A$, then it results $\left\|C_{m_{h}, s_{h}}\right\|_{\infty} \leq 2^{s_{h}}-1$ and hence (7.74) immediately follows by (7.72) and (7.73). Moreover, (7.75) can be deduced with the same arguments in [33], taking into account estimate (3.29). 
Remark 7.3. From the previous estimate, it is possible to deduce the rate of convergence of the approximation according to the smoothness properties of $f \in C(\mathrm{~S})$. For instance, if $f_{y} \in W_{r_{1}}$ and $f_{x} \in W_{r_{2}}$, uniformly w.r.t. $y$ and $x$ respectively, then choosing $s_{1} \geq \frac{r_{1}}{2}$ and $s_{2} \geq \frac{r_{2}}{2}$, by (2.10), we immediately deduce that

$$
\left\|f-\mathbf{B}_{\mathbf{m}, \mathbf{s}} f\right\|_{\mathrm{S}} \leq \mathcal{C}\left\{\sup _{y \in[0,1]}\left[\frac{\left\|f_{y}\right\|_{W_{r_{1}}}}{\sqrt{m_{1}^{r_{1}}}}+\frac{\left\|f_{y}\right\|}{m_{1}^{s_{1}}}\right]+\sup _{x \in[0,1]}\left[\frac{\left\|f_{x}\right\|_{W_{r_{2}}}}{\sqrt{m_{2}^{r_{2}}}}+\frac{\left\|f_{x}\right\|}{m_{2}^{s_{2}}}\right]\right\} .
$$

Estimate (7.76) suggests that when the smoothness of the function $f$ is different w.r.t the two variables, then it is possible to obtain a significant reduction in the computational cost, both in terms of function samples and in the construction of matrices $C_{m_{h}, s_{h}}, h=1,2$, with respect to the case $m_{1}=m_{2}$.

7.2. A cubature rule. The above introduced $\mathbf{B}_{\mathbf{m}, \mathbf{s}}$ operator can be usefully employed in the numerical cubature. Indeed for integrals of the type $\int_{\mathrm{S}} f(x, y) d x d y$, it is possible to deduce the following cubature rule

$$
\begin{aligned}
\int_{\mathrm{S}} f(x, y) d x d y & =\int_{\mathrm{S}} \mathbf{B}_{\mathbf{m}, \mathbf{s}} f(x, y) d x d y+\mathbf{R}_{\mathbf{m}, \mathbf{s}} f \\
& =: \boldsymbol{\Sigma}_{\mathbf{m}, \mathbf{s}} f+\mathbf{R}_{\mathbf{m}, \mathbf{s}} f .
\end{aligned}
$$

By (7.73) and taking into account that

$$
\int_{0}^{1} p_{m, k}(t) d t=\frac{1}{m+1}, \quad k \in N_{0}^{m}
$$

it is not hard to prove that

$$
\mathbf{\Sigma}_{\mathbf{m}, \mathbf{s}} f=\sum_{i=0}^{m_{1}} \sum_{j=0}^{m_{2}} \mathbf{T}_{i, j}^{(\mathbf{s})} f\left(t_{i}, t_{j}\right),
$$

where for any $(i, j) \in N_{0}^{m_{1}} \times N_{0}^{m_{2}}$,

$$
\mathbf{T}_{i, j}^{(\mathbf{s})}=\frac{1}{\left(m_{1}+1\right)\left(m_{2}+1\right)}\left(\sum_{r=0}^{m_{1}}\left(C_{m_{1}, s_{1}}\right)_{r, i}\right)\left(\sum_{k=0}^{m_{2}}\left(C_{m_{2}, s_{2}}\right)_{k, j}\right) .
$$

The previous rule for $m_{1}=m_{2}$ and $s_{1}=s_{2}$ was introduced in [33]. The stability and convergence of the cubature rule are stated in the next theorem which can be obtained as a direct application of (7.75).

Theorem 7.11. With the notation used in (7.77)-(7.78) and for any $f \in C(\mathrm{~S})$, the cubature formula is convergent, holding

$$
\begin{aligned}
\left|\mathbf{R}_{\mathbf{m}, \mathbf{s}} f\right| & \leq \mathcal{C}\left\{\sup _{y \in[0,1]}\left[\omega_{\varphi}^{2 s_{1}}\left(f_{y}, \frac{1}{\sqrt{m_{1}}}\right)+\frac{\left\|f_{y}\right\|}{m_{1}^{s_{1}}}\right]\right. \\
& \left.+\sup _{x \in[0,1]}\left[\omega_{\varphi}^{2 s_{2}}\left(f_{x}, \frac{1}{\sqrt{m_{2}}}\right)+\frac{\left\|f_{x}\right\|}{m_{2}^{s_{2}}}\right]\right\}, \quad \mathcal{C} \neq \mathcal{C}(\mathbf{m}, f), \quad \mathcal{C}=\mathcal{C}(\mathbf{s})
\end{aligned}
$$

and numerically stable, i.e.,

$$
\sup _{m_{1}} \sup _{m_{2}} \sum_{i=0}^{m_{1}} \sum_{j=0}^{m_{2}}\left|\mathbf{T}_{i, j}^{(\mathbf{s})}\right|<\infty \text {. }
$$


Remark 7.4. As for the generalized Bernstein approximation, also in this case from estimate (7.79), it is possible to deduce suitable convergence order for specific classes of functions. For instance for functions that are in Sobolev spaces w.r.t the single variable and estimate like (7.76) can be obtained also for $\mathbf{R}_{\mathbf{m}, \mathbf{s}} f$. In addition taking into account the different estimates w.r.t the single variables of $f$, it is possible to construct, with the same attained error, a cubature rule with a computational saving respect both the computation of the function samples and the construction of the weights of the rule.

Now, we want to give just two examples of the proposed cubature formula. In both cases, the value of the integral is computed in machine precision by means of a Gaussian cubature formula obtained as a tensor product of two Gauss-Legendre rules.

Example 7.4. Consider the double integral

$$
\int_{0}^{1} \int_{0}^{1} \cos (x y) e^{|y-0.2|^{\frac{17}{3}}} d x d y \sim 9.779542891104441 e-01 .
$$

The integrating function is in $W_{r_{1}}$ for any integer $r_{1}$ with respect to the variable $x$, while is in $W_{5}$ with respect to the variable $y$. Therefore from (7.79), we get that the order of convergence of the formula is $\mathcal{O}\left(m^{-5 / 2}\right)$. Nevertheless, it is clear that $m_{1}$ and $s_{1}$ could be taken reasonably small while $m_{2}$ has to increase in order to get an high number of correct digits. Therefore we fixed $m_{1}=64, s_{1}=1024$, while for any value of $m_{2}$ we consider different values for $s_{2}$. The results are shown in the following Table 5

\begin{tabular}{|c|l|l|l|l|l|l|}
\hline$m_{2}$ & $s_{2}=4$ & $s_{2}=8$ & $s_{2}=16$ & $s_{2}=32$ & $s_{2}=64$ & $s_{2}=128$ \\
\hline 64 & $6.31 e-07$ & $7.04 e-08$ & $1.86 e-08$ & $6.69 e-09$ & $2.86 e-09$ & $1.38 e-09$ \\
\hline 128 & $6.04 e-08$ & $2.79 e-09$ & $4.18 e-10$ & $9.29 e-11$ & $2.57 e-11$ & $8.39 e-12$ \\
\hline 256 & $6.22 e-09$ & $1.05 e-10$ & $8.00 e-12$ & $1.01 e-12$ & $1.63 e-13$ & $3.13 e-14$ \\
\hline 512 & $6.89 e-10$ & $4.01 e-12$ & $1.38 e-13$ & $8.10 e-15$ & $7.77 e-16$ & $1.32 e-15$ \\
\hline 1024 & $8.05 e-11$ & $1.63 e-13$ & $7.77 e-16$ & $9.99 e-16$ & $1.33 e-15$ & $1.44 e-15$ \\
\hline
\end{tabular}

TABLE 5. Absolute errors for Example 7.4

As the table shows, the numerical behavior in surely better than the theoretical estimate predicts. Moreover, it is possible to show that increasing $m_{1}$ does not lead to any relevant improvement in the exact digits in the results.

Example 7.5. Consider the double integral

$$
\int_{0}^{1} \int_{0}^{1} e^{(x+y)^{2}}|\sin (y)-0.5|^{\frac{7}{2}} d x d y \sim 4.8794503105779 e-02 .
$$

The integrating function is in $W_{r_{1}}$ for any integer $r_{1}$ with respect to the variable $x$ while is in $W_{3}$ with respect to the variable $y$. Therefore from (7.79), we get that the order of convergence of the formula is $\mathcal{O}\left(m^{-3 / 2}\right)$. As in the previous example, we fix $m_{1}$ and $s_{1}$, while $m_{2}$ is taken increasing in order to get an high number of correct digits. Therefore we fixed $m_{1}=256, s_{1}=4096$, while for any value of $m_{2}$ we consider different values for $s_{2}$. The results are shown in the following Table 6.

Also in this case, if we take higher values for $m_{1}$, no benefits can be found on the obtained results. Moreover also here it is evident that for a fixed value of $m_{2}$, the choice of higher values for $s_{2}$ leads to gain more exact digits, till some threshold $\widetilde{s}_{2}$ depending on $m_{2}$ (see for instance the results for $m_{2}=256$ and $s_{2}=32,64,128$ or $m_{2}=512$ and $\left.s_{2}=16,32,64,128\right)$. 


\begin{tabular}{|c|l|l|l|l|l|l|}
\hline$m_{2}$ & $s_{2}=4$ & $s_{2}=8$ & $s_{2}=16$ & $s_{2}=32$ & $s_{2}=64$ & $s_{2}=128$ \\
\hline 64 & $1.14 e-06$ & $6.53 e-08$ & $8.69 e-09$ & $1.48 e-09$ & $1.90 e-10$ & $6.92 e-11$ \\
\hline 128 & $1.18 e-07$ & $2.57 e-09$ & $1.69 e-10$ & $2.19 e-11$ & $8.05 e-12$ & $6.62 e-12$ \\
\hline 256 & $1.31 e-08$ & $1.05 e-10$ & $3.08 e-12$ & $4.11 e-13$ & $2.85 e-13$ & $2.79 e-13$ \\
\hline 512 & $1.53 e-09$ & $4.72 e-12$ & $5.92 e-14$ & $1.22 e-14$ & $1.11 e-14$ & $1.11 e-14$ \\
\hline 1024 & $1.84 e-10$ & $2.35 e-13$ & $1.41 e-15$ & $5.55 e-16$ & $5.55 e-16$ & $5.55 e-16$ \\
\hline
\end{tabular}

TABLE 6. Absolute errors for Example 7.5

7.3. Fredholm integral equations on the square. In this section, we want to show a possible application of the cubature rule introduced above to the numerical approximation of the solution of a Fredholm integral equation (FIE) defined on S . For the sake of simplicity, we will limit ourselves to the case $m_{1}=m_{2}$ and $s_{1}=s_{2}$, but all the results, mutatis mutandis can be obtained in the more general case.

First of all, we introduce the following bivariate Sobolev-type space

$$
\mathbf{W}_{r}=\left\{f \in C(\mathrm{~S}): \mathcal{M}_{r} f:=\max \left\{\max _{(x, y) \in \mathrm{S}}\left|f_{y}^{(r)}(x) \varphi^{r}(x)\right|, \max _{(x, y) \in \mathrm{S}}\left|f_{x}^{(r)}(y) \varphi^{r}(y)\right|\right\}<\infty\right\},
$$

where the superscript $(r)$ denotes the $r$-th derivative of the one-dimensional function $f_{y}$ or $f_{x}$ and the function $\varphi(z)=\sqrt{z(1-z)}$. $\mathbf{W}_{r}$ will be equipped with the norm $\|f\|_{\mathbf{W}_{r}}=\|f\|_{\mathrm{S}}+\mathcal{M}_{r} f$.

Let us consider the following bivariate FIE on the square $\mathrm{S}$

$$
f(x, y)-\mu \int_{\mathrm{S}} f(z, t) k(x, y, z, t) d z d t=g(x, y),(x, y) \in \mathrm{S},
$$

where $\mu \in \mathbb{R}, k$ defined on $\mathrm{S} \times \mathrm{S}$ and $g$ defined on $\mathrm{S}$ are given functions, while $f$ is the unknown function. Denoting by

$$
\mathbf{K} f(x, y)=\mu \int_{\mathrm{S}} k(x, y, z, t) f(z, t) d z d t
$$

(7.82) can be rewritten in operatorial form as

$$
(\mathbf{I}-\mathbf{K}) f=g,
$$

where $\mathbf{I}$ is the identity operator on $C(\mathrm{~S})$. Here and in the sequel, we will denote $k_{(z, t)}$ for meaning that the function of four variables $k$ is considered as a function of the only pair $(x, y)$.

Using standard arguments, it is possible to prove that if $k(x, y, z, t)$ is continuous, then $\mathbf{K}$ : $C(\mathrm{~S}) \rightarrow C(\mathrm{~S})$ is compact and consequently the Fredholm Alternative holds true for (7.83) in $C(\mathrm{~S})$ (see for instance [6]). Moreover if for some $r \in \mathbb{N}$,

$$
\sup _{(z, t) \in \mathrm{S}}\left\|k_{(z, t)}\right\|_{W_{r}}<+\infty
$$

then $\mathbf{K} f \in W_{r}$ for any $f \in C(\mathrm{~S})$.

Starting with the cubature rule (7.78) written with $m_{1}=m_{2}=: m$ and $s_{1}=s_{2}=: s$, we can define the following discrete operator

$$
\mathbf{K}_{m} f(x, y)=\mu \sum_{i=0}^{m} \sum_{j=0}^{m} \mathbf{T}_{i, j}^{(\mathbf{s})} k\left(x, y, t_{i}, t_{j}\right) f\left(t_{i}, t_{j}\right)
$$

and consider the operator equation

$$
\left(\mathbf{I}-\mathbf{K}_{m}\right) f_{m}=g,
$$


where $f_{m}$ is unknown. Collocating on the pairs $\left(t_{h}, t_{\ell}\right),(h, \ell) \in N_{0}^{m} \times N_{0}^{m}$, the quantities $\beta_{i j}=f\left(\mathbf{P}_{i, j}\right),(i, j) \in N_{0}^{m} \times N_{0}^{m}$, come out to be the unknowns of the linear system

$$
\beta_{h \ell}-\mu \sum_{i=0}^{m} \sum_{j=0}^{m} \mathbf{T}_{i, j}^{(s)} k\left(t_{h}, t_{\ell}, t_{i}, t_{j}\right) \beta_{i j}=g\left(t_{h}, t_{\ell}\right), \quad(h, \ell) \in N_{0}^{m} \times N_{0}^{m} .
$$

The matrix solution $\left(\beta_{i j}^{*}\right)_{i, j=0,1 \ldots, m}$ of this system, if it exists, allows us to construct the Nyström interpolant in two variables

$$
f_{m}(x, y)=\mu \sum_{i=0}^{m} \sum_{j=0}^{m} \mathbf{T}_{i, j}^{(s)} k\left(x, y, t_{i}, t_{j}\right) \beta_{i j}^{*}+g(x, y)
$$

which will approximate the unknown $f$. Now, denote by $\boldsymbol{\Gamma}_{m, s}$ the coefficient matrix of system (7.86), which is a $(m+1)$ block matrix, the entries of which are matrices of order $m+1$.

Denoting by cond $\left(\boldsymbol{\Gamma}_{m, s}\right)$ the condition number in infinity norm of $\boldsymbol{\Gamma}_{m, s}$, the following theorem holds true (see [33]).

Theorem 7.12. Assume that $k$ is continuous w.r.t. its four variables and that $\operatorname{Ker}\{\mathbf{I}-\mathbf{K}\}=\{0\}$ in $C(\mathrm{~S})$. Denote by $f$ the unique solution of $(7.85)$ in $C(\mathrm{~S})$ for a given $g \in C(\mathrm{~S})$. If in addition, for some $r \in \mathbb{N}, k$ satisfies (7.84), $\mathbf{g} \in \mathbf{W}_{r}$, and

$$
\sup _{(x, y) \in \mathrm{S}}\left\|k_{(x, y)}\right\| \mathbf{W}_{r}<+\infty
$$

then, for $m$ sufficiently large, the system (7.86) is uniquely solvable and well-conditioned too, since

$$
\operatorname{cond}\left(\boldsymbol{\Gamma}_{m, s}\right) \leq \mathcal{C}, \quad \mathcal{C} \neq \mathcal{C}(m), \quad \mathcal{C}=\mathcal{C}(s) .
$$

Moreover, for any $s \geq \frac{r}{2}$, there results

$$
\left\|f-f_{m}\right\|_{\mathrm{S}} \leq \mathcal{C} \frac{\|f\|_{\mathbf{W}_{r}}}{\sqrt{m^{r}}}
$$

where $\mathcal{C} \neq \mathcal{C}(m, f)$ and $\mathcal{C}=\mathcal{C}(s)$

Remark 7.5. Several computational details and numerical tests about the proposed Nyström method can be found in [33]. In particular, in that paper the case in which the function kernel $k(x, y, z, t)$ shows some symmetry was discussed. Indeed, the symmetry properties of the kernel are inherited by the matrix $\boldsymbol{\Gamma}_{m, s}$. And this fact could be useful for reducing the computational effort in solving the linear system (7.86).

\section{Computational Details}

8.1. On the computation of $B_{m, s} f$. In order to construct $B_{m, s} f$ as defined in (3.27), we give some computational details about the matrix $C_{m, s}$ [33]. Such matrix is defined by the matrix $A$ in (3.25) which can be constructed by rows by making use of the triangular scheme in (2.4). In this way, for each row, $m^{2}$ long operations are required. On the other hand, since $A$ is centrosymmetric, i.e., $A=J A J$, where $J$ is the counter-identity matrix of order $m+1$ (i.e., $J_{i, j}=\delta_{i, m-j}, \forall i, j \in N_{0}^{m}$, being $\delta_{h, k}$ the Kronecker delta), it will be enough to compute only the first $\left(\frac{m+1}{2}\right)$ rows for odd $m$, or $\left(\frac{m+2}{2}\right)$ rows, when $m$ is even. Therefore, the construction of $A$ requires about $\frac{m^{3}}{2}$ long operations. Furthermore, since the product of two centrosymmetric matrices can be performed in almost $\frac{m^{3}}{4}$ long operations [1], the matrix $C_{m, s}$ in (3.24) can be constructed in almost $(s-2) \mathrm{m}^{3} / 4$ long operations, instead of $(s-2) m^{3}$ ones, i.e., with a saving 
of about the $75 \%$. A more significant reduction is achieved when the parameter $s=2^{p}, p \in N$. Indeed, by using [32, (14)]

$$
C_{m, 2^{p}}=C_{m, 2^{p-1}}+(I-A)^{2^{p-1}} C_{m, 2^{p-1}},
$$

that allow to the following relation among the polynomials

$$
B_{m, 2^{p}} f(x)=2 B_{m, 2^{p-1}} f(x)-B_{m, 2^{p-1}}^{2} f(x),
$$

the matrix $C_{m, s}$ can be determined by $2\left(\log _{2} s-1\right)$ products of centrosymmetric matrices and therefore requiring almost $\frac{m^{3}}{2}\left(\log _{2} s-1\right)$ long operations. For instance, for $s=256$, if we use definition (3.24), then we have 255 products of centrosymmetric matrices that require about $255 \frac{\mathrm{m}^{3}}{4} \sim 63.7 \mathrm{~m}^{3}$ long operations. On the contrary, if we use (8.90), then approximatively only $3.5 \mathrm{~m}^{3}$ long operations are needed.

8.2. Computation of the derivatives of $B_{m, s} f$. As regards the first derivative of the Bernstein polynomials $B_{m, s} f$, by (3.27), we obtain the following useful representation

$$
\left(B_{m, s} f\right)^{\prime}(x)=\mathbf{p}_{m}^{1}(x) C_{m, s} \mathbf{f}_{m}
$$

where $\mathbf{f}_{m}$ was defined in (3.28) and we set

$$
\mathbf{p}_{m}^{1}(x):=\left[p_{m, 0}^{\prime}(x), \ldots, p_{m, m}^{\prime}(x)\right]
$$

where

$$
p_{m, k}^{\prime}(x)=m\left(p_{m-1, k-1}(x)-p_{m-1, k}(x)\right), \quad k \in \mathbb{N}_{0}^{m},
$$

with the usual convention $p_{m, j}(x)=0$ if $j \notin \mathbb{N}_{0}^{m}$.

8.3. Coefficients of the quadrature rules for Hilbert and Hadamard transforms. The coefficients of the rule (6.59) take the following expression

$$
D_{m, j}^{(s)}(t)=\sum_{i=0}^{m}\left(C_{m, s}\right)_{i, j} \int_{0}^{1} \frac{p_{m, i}(x)-p_{m, i}(t)}{x-t} d x=: \sum_{i=0}^{m}\left(C_{m, s}\right)_{i, j} q_{m, i}(t),
$$

where the polynomials $q_{m, i}(t)$ can be computed via recurrence relation as stated in the following proposition.

Proposition 8.1. For all $m \in \mathbb{N}, m>1$, the polynomials $q_{m, k}(t)$, with $k \in \mathbb{N}_{0}^{m}$, satisfy the following recurrence relation

$$
\begin{aligned}
q_{0,0}(t) & =0, \quad q_{1,0}(t)=-1, \quad q_{1,1}(t)=1, \\
q_{m, 0}(t) & =(1-t) q_{m-1,0}(t)-\frac{1}{m}, \\
q_{m, k}(t) & =(1-t) q_{m-1, k}(t)+t q_{m-1, k-1}(t), \quad 1 \leq k \leq m-1, \\
q_{m, m}(t) & =t q_{m-1, m-1}(t)+\frac{1}{m} .
\end{aligned}
$$


Proof. For $1 \leq k \leq m-1$, by using recurrence relation (2.4) and taking into account that $\int_{0}^{1} p_{m, k}(x) d x=\frac{1}{m+1}$ for all $k \in \mathbb{N}_{0}^{m}$, we get

$$
\begin{aligned}
q_{m, k}(t) & =\int_{0}^{1} \frac{(1-x) p_{m-1, k}(x)-(1-t) p_{m-1, k}(t)}{x-t} d x \\
& +\int_{0}^{1} \frac{x p_{m-1, k-1}(x)-t p_{m-1, k-1}(t)}{x-t} d x \\
& =q_{m-1, k}(t)-\int_{0}^{1} \frac{x p_{m-1, k}(x)-t p_{m-1, k}(t)}{x-t} d x \\
& +\int_{0}^{1} \frac{x p_{m-1, k-1}(x)-t p_{m-1, k-1}(t)}{x-t} d x \\
& =q_{m-1, k}(t)-\frac{1}{m}-t q_{m-1, k}(t)+\frac{1}{m}+t q_{m-1, k-1}(t) \\
& =(1-t) q_{m-1, k}(t)+t q_{m-1, k-1}(t) .
\end{aligned}
$$

For $k=0$,

$$
\begin{aligned}
q_{m, 0}(t) & =\int_{0}^{1} \frac{(1-x) p_{m-1,0}(x)-(1-t) p_{m-1,0}(t)}{x-t} d x=q_{m-1,0}(t)-\frac{1}{m}-t q_{m-1,0}(t) \\
& =(1-t) q_{m-1,0}(t)-\frac{1}{m} .
\end{aligned}
$$

For $k=m$, we proceed in the same way.

\section{Setting}

$$
\mathbf{q}_{m}(t)=\left[q_{m, 0}(t), q_{m, 1}(t), \ldots, q_{m, m}(t)\right],
$$

the quadrature rule (6.59) can be rewritten as

$$
\mathcal{F}_{m, s} f(t)=\mathbf{q}_{m}(t) C_{m, s} \mathbf{f}_{m} .
$$

Moreover, the quadrature rule $\mathcal{H}_{m, s}$ in (6.63) tales the form

$$
\mathcal{H}_{m, s} f(t)=\left[\mathbf{q}_{m}(t)+\log \left(\frac{1-t}{t}\right) \mathbf{p}_{m}(t)\right] C_{m, s} \mathbf{f}_{m} .
$$

About the coefficients of the formula (6.60), by (6.59) and (8.94), we get $\forall j \in \mathbb{N}_{0}^{m}$,

$$
\bar{D}_{m, j}^{(s)}(t)=\sum_{i=0}^{m}\left(C_{m, s}\right)_{i, j} d_{m, i}(t), \quad d_{m, i}(t):=q_{m, i}^{\prime}(t),
$$

where the polynomials $d_{m, i}(t), i=0, \ldots, m$ can be computed recursively according to next proposition which easily follows by Proposition 8.1 .

Proposition 8.2. For all $m \in \mathbb{N}, m>1$, the polynomials $d_{m, k}(t), k \in \mathbb{N}_{0}^{m}$, satisfy the following recurrence relation

$$
\begin{aligned}
d_{1,0}(t) & =0, \quad d_{1,1}(t)=0, \\
d_{m, 0}(t) & =(1-t) d_{m-1,0}(t)-q_{m-1,0}(t), \\
d_{m, k}(t) & =(1-t) d_{m-1, k}(t)-q_{m-1, k}(t)+t d_{m-1, k-1}(t)+q_{m-1, k-1}(t), \quad 0<k<m, \\
d_{m, m}(t) & =t d_{m-1, m-1}(t)+q_{m-1, m-1}(t) .
\end{aligned}
$$




\section{Setting}

$$
\mathbf{d}_{m}(t)=\left[d_{m, 0}(t), d_{m, 1}(t), \ldots, d_{m, m}(t)\right],
$$

the quadrature rule (6.60) takes the following form

$$
\mathcal{F}_{m, s}^{1} f(t)=\mathbf{d}_{m}(t) C_{m, s} \mathbf{f}_{m} .
$$

Finally by (8.99), (3.27) and (8.92), the rule $\mathcal{H}_{m, s}^{1}$ defined in (6.64) takes the following vectorial form

$$
\mathcal{H}_{m, s}^{1} f(t)=\left[\mathbf{d}_{m}(t)+\log \left(\frac{1-t}{t}\right) \mathbf{p}_{m}^{1}(t)-\frac{2}{t(1-t)} \mathbf{p}_{m}(t)\right] C_{m, s} \mathbf{f}_{m} .
$$

\section{ACKNOWLEDGEMENTS}

The Authors would like to thank the Referees for their helpful comments and suggestions that certainly improved the quality of the manuscript.

\section{MEMBERSHIPS}

The Authors are members of the INdAM-GNCS Research Group and of the TAA-UMI Research Group.

This research has been accomplished within the RITA "Research ITalian network on Approximation" and the GNCS 2020 project "Approssimazione multivariata ed equazioni funzionali per la modellistica numerica".

\section{REFERENCES}

[1] I. T. Abu-Jeib: Algorithms for Centrosymmetric and Skew-Centrosymmetric Matrices, Missouri J. Math. Sci., 18 (1) (2006), 1-8.

[2] F. Altomare, M. Campiti: Korovkin-type approximation theory and its applications, De Gruyter Studies in Mathematics, 17, Walter de Gruyter \& C., Berlin (1994).

[3] F. Altomare, M. Cappelletti Montano, V. Leonessa and I. Rasa: Markov operators, positive semigroups and approximation processes, De Gruyter Studies in Mathematics 61, De Gruyter, Berlin (2014).

[4] P. N. Agrawal, H. S. Kasana: On iterative combinations of Bernstein polynomials, Demonstr. Math. 17, (1984) 777-783.

[5] U. Amato, B. Della Vecchia: Bridging Bernstein and Lagrange polynomials, Math. Commun., 20 (2) (2015), 151-160.

[6] K. E. Atkinson: The Numerical Solution of Integral Equations of the second kind, Cambridge Monographs on Applied and Computational Mathematics, 4. Cambridge University Press, Cambridge (1997).

[7] H. Brass, J. W. Fischer: Error bounds for Romberg quadrature, Numer. Math., 82 (3) (1999), 389-408.

[8] M. Campiti: Convergence of Iterated Boolean-type Sums and Their Iterates, Numer. Funct. Anal. Optim., 39 (10) (2018), 1054-1063.

[9] M. R. Capobianco, G. Mastroianni and M. G. Russo: Pointwise and uniform approximation of the finite Hilbert transform, "Approximation and Optimization, Proceedings of International Conference on Approximation and Optimization (ICAOR) Cluj-Napoca, July 29-August 1, 1996, (Eds. Stancu D. Coman G., Breckner W.W., Blaga P.) 1 (1997), 45-66.

[10] S. Cooper, S. Waldron: The eigenstructure of the Bernstein operator, J. Approx. Theory, 105 (1) (2000), 133-165.

[11] P. J. Davis, P. Rabinowitz: Methods of numerical integration, Computer Science and Applied Mathematics, Academic Press Inc., Orlando, FL (1984).

[12] M. C. De Bonis, G. Mastroianni: Projection methods and condition numbers in uniform norm for Fredholm and Cauchy singular integral equations, SIAM J. Numer. Anal., 44 (4) (2006), 1-24.

[13] Z. Ditzian, V. Totik: Moduli of smoothness, Springer Series in Computational Mathematics 9, Springer-Verlag, New York (1987).

[14] Z. Ditzian, V. Totik: Remarks on Besov spaces and best polynomial approximation, Proc. Amer. Math. Soc., 104 (4) (1988).

[15] B. R. Draganov: Strong estimates of the weighted simultaneous approximation by the Bernstein and Kantorovich operators and their Boolean sums, J. Approx. Theory, 200 (2015), 92-135.

[16] G. Farin: Curves and surfaces for computer aided geometric design. A practical guide, Third edition. Computer Science and Scientific Computing. Academic Press, Inc., Boston, MA (1993)

[17] G. Felbecker: Linearkombinationen von iterierten Bernsteinoperatoren, Manuscripta Math., 29 (2-4) (1979), 229-246. 
[18] F. Filbir, D. Occorsio and W. Themistoclakis: Approximation of Finite Hilbert and Hadamard transforms by using equally spaced nodes, Mathematics, 8 (4) (2020), Article number 542.

[19] H. H. Gonska, X. L. Zhou: Approximation theorems for the iterated Boolean sums of Bernstein operators, J. Comput. Appl. Math., 53 (1994) 21-31.

[20] P. Junghanns, U. Luther: Cauchy singular integral equations in spaces of continuous functions and methods for their numerical solution, ROLLS Symposium (Leipzig, 1996). J. Comput. Appl. Math., 77 (1-2) (1997), 201-237.

[21] A. I. Kalandiya: Mathematical Methods of Two-Dimensional Elasticity, Publ., Nauka Moscow (1973).

[22] F. King: Hilbert Transforms, I \& II. Cambridge University Press, Cambridge (2009).

[23] G. G. Lorentz: Bernstein polynomials, Second edition. Chelsea Publishing Co., New York (1986)

[24] G. Mastroianni, M. R. Occorsio: Una generalizzazione dell'operatore di Bernstein, Rend. dell'Accad. di Scienze Fis. e Mat. Napoli (Serie IV), 44 (1977), 151-169

[25] G. Mastroianni, M. R. Occorsio: Alcuni algoritmi per il calcolo numerico di integrali a valor principale secondo Cauchy, (available in Italian only) Rapporto Tecnico I.A.M. 3/84.

[26] G. Mastroianni, M.R. Occorsio: An algorithm for the numerical evaluation of a Cauchy principal value integral, Ricerche Mat., 33 (1) (1984), 3-18.

[27] G. Mastroianni, M. G. Russo and W. Themistoclakis: Numerical Methods for Cauchy Singular Integral Equations in Spaces of Weighted Continuous Functions, Operator Theory Advances and Applications, 160, Birkhäuser Verlag Basel, Switzerland (2005), 311-336.

[28] G. Mastroianni, M. G. Russo and W. Themistoclakis: The boundedness of the Cauchy singular integral operator in weighted Besov type spaces with uniform norms, Integr. Equ. Oper. Theory, 42, Birkhäuser Verlag Basel (2002), 57-89.

[29] G. Mastroianni, W. Themistoclakis: A numerical method for the generalized airfoil equation based on the de la Vallée Poussin interpolation, J. Comput. Appl. Math., 180 (2005), 71-105.

[30] C. Micchelli: The saturation class and iterates of Bernstein polynomials, J. Approx. Th., 8 (1973), 1-18

[31] G. Monegato: Numerical evaluation of hypersingular integrals, J. Comp. Appl. Math., 50 (1994), 9-31.

[32] D. Occorsio, M. G. Russo: A Nyström method for Fredholm integral equations based on equally spaced knots, Filomat, 28 (1) (2014), 49-63.

[33] D. Occorsio, M. G. Russo: Bivariate Generalized Bernstein Operators and their application to Fredholm Integral Equations, Publ. Inst. Math. N. S., 100 (114) (2016), 141-162.

[34] D. Occorsio, A. C. Simoncelli: How to go from Bézier to Lagrange curves by means of generalized Bézier curves, Facta Univ. Ser. Math. Inform. (Niš), 11 (1996), 101-111.

[35] D. Occorsio: Some new properties of Generalized Bernstein polynomials, Stud. Univ. Babeş-Bolyai Math, 56 (3) (2011), 147-160.

[36] I. Rasa: Iterated Boolean sums of Bernstein and related operators, Rev. Anal. Numér. Théor. Approx, 35 (2006), 111-115.

[37] S. Weiwei, W. Jiming: Interpolatory quadrature rules for Hadamard finite-part integrals and their superconvergence, IMA J. Numer. Anal., 28 (2008), 580-597.

[38] G. Tachev: From Bernstein polynomials to Lagrange interpolation, Proceedings of 2nd International Conference on Modelling and Development of Intelligent Systems (MDIS 2011), (2011), 192-197.

\author{
DONATELLA OCCORSIO \\ UNIVERSITY OF BASILICATA \\ DePARTMENT OF MATHEMATICS, COMPUTER SCIENCE AND ECONOMICS \\ Viale Dell'Ateneo LuCANO, 10, 85100, PotenZa, ItAly \\ ORCID: 0000-0001-9446-4452 \\ E-mail address: donatella.occorsio@unibas.it \\ MARIA GRAZIA RUSSO \\ UNIVERSITY OF BASILICATA \\ Department OF MATHEMATICS, COMPUTER SCIENCE AND ECONOMICS \\ VIALE DELL'ATENEO LuCANO, 10, 85100, PotenZA, ITALY \\ ORCID: 0000-0002-4078-620X \\ E-mail address: mariagrazia.russo@unibas.it
}

WOULA THEMISTOCLAKIS

C.N.R. NATIONAL RESEARCH COUNCIL OF ITALY

IAC INSTITUTE FOR APPLIED COMPUTING " MAURo PiCONE"

VIA P. CASTELLINO, 111, NAPLES, 80131, ITALY

ORCID: 0000-0002-6185-1154

E-mail address: woula.themistoclakis@enr. it 